\title{
On the existence of weak solutions for singular strongly nonlinear boundary value problems on the half-line
}

\author{
Stefano Biagi ${ }^{1}$
}

Received: 4 April 2019 / Accepted: 3 August 2019 / Published online: 16 August 2019

(c) Fondazione Annali di Matematica Pura ed Applicata and Springer-Verlag GmbH Germany, part of Springer Nature 2019

\section{Abstract}

In the present paper, we consider boundary value problems on the real half-line $\Lambda:=[0, \infty)$ of the following form

$$
\left\{\begin{array}{l}
\left(\Phi\left(a(t, x(t)) x^{\prime}(t)\right)\right)^{\prime}=f\left(t, x(t), x^{\prime}(t)\right) \text { a.e. on } \Lambda, \\
x(0)=v_{1}, \quad x(\infty)=v_{2},
\end{array}\right.
$$

where $\Phi: \mathbb{R} \rightarrow \mathbb{R}$ is a strictly increasing homeomorphism, $a \in C(\Lambda \times \mathbb{R}, \mathbb{R})$ is nonnegative which can vanish on a set of zero Lebesgue measure and $f$ is a Caratheódory function on $\Lambda \times \mathbb{R}^{2}$. Under very general assumptions on the functions $a$ and $f$, including an appropriate version of the well-known Nagumo-Wintner growth condition, we prove the existence of at least one solution of the above problem in a suitable Sobolev space. Our approach combines a fixed-point technique with the method of lower/upper solutions.

Keywords Singular ODEs · Heteroclinic solutions $\cdot \Phi$-Laplace operators · Nonlinear ODEs $\cdot$ BVPs on unbounded intervals $\cdot$ Nagumo-Wintner condition

Mathematics Subject Classification 34C37 $\cdot 34 \mathrm{~B} 16 \cdot 34 \mathrm{~B} 40 \cdot 34 \mathrm{~L} 30$

\section{Introduction}

The main aim of the present paper is to obtain existence results for boundary value problems (BVPs, in the sequel) on the half-line $\Lambda:=[0, \infty)$ associated with strongly nonlinear, non-autonomous ordinary differential equations (ODEs, for short) involving the so-called $\Phi$ Laplace operator on $\mathbb{R}$. To be more precise, our main result Theorem 1 ensures the existence of at least one solution (in a suitable weak sense) of BVPs of the following form

$\bowtie \quad$ Stefano Biagi

biagi@dipmat.univpm.it

1 Università Politecnica della Marche, Via Brecce Bianche, 60131 Ancona, Italy 


$$
\left\{\begin{array}{l}
\left(\Phi\left(a(t, x(t)) x^{\prime}(t)\right)\right)^{\prime}=f\left(t, x(t), x^{\prime}(t)\right) \text { a.e. on } \Lambda, \\
x(0)=v_{1}, \quad x(\infty)=v_{2},
\end{array}\right.
$$

where $\Phi: \mathbb{R} \rightarrow \mathbb{R}$ is a generic strictly increasing homeomorphism (the $\Phi$-Laplace operator), $f: \Lambda \times \mathbb{R}^{2} \rightarrow \mathbb{R}$ is a Caratheódory function and $v_{1}, \nu_{2} \in \mathbb{R}$. As for the function $a$, we assume that $a \in C(\Lambda \times \mathbb{R}, \mathbb{R})$ is nonnegative and

$$
|\{(t, x): a(t, x)=0\}|=0
$$

(here and throughout, $|A|$ stands for the Lebesgue measure of a subset $A$ of some Euclidean space). As a consequence of (2), the ODE

$$
\left(\Phi\left(a(t, x(t)) x^{\prime}(t)\right)\right)^{\prime}=f\left(t, x(t), x^{\prime}(t)\right)
$$

may be singular; due to this fact, it seems quite natural to look for solutions in the Sobolev space $W_{\text {loc }}^{1,1}(\Lambda)$, rather than in the space $C^{1}(\Lambda, \mathbb{R})$.

Differential equations of the form (3) intervene in several models arising, e.g., in nonNewtonian fluid theory, nonlinear elasticity, theory of capillary surfaces and diffusion of flows in porous media (see $[4,15,21]$ and the references therein); a concrete example of this fact is given by the following problem,

$$
\left\{\begin{array}{l}
\left(|x(t)|^{p-1} x^{\prime}(x)\right)^{\prime}=\lambda f\left(x^{1 / q}\right), \quad \text { for } t \in[-1,1], \\
x(-1)=a, \quad x(1)=b,
\end{array}\right.
$$

proposed by Bobisud in [4] as a model for one-dimensional turbulent flow of a polytropic gas in a porous medium. We explicitly observe that the ODE appearing in this model is a very particular case of (3), in which one takes

$$
f\left(t, x, x^{\prime}\right)=\lambda f\left(x^{1 / q}\right), \quad a(t, x) \equiv 1 \quad \text { and } \quad \Phi(z)=|z|^{p-1} z .
$$

In view of their several applications, ODEs of the form (3) have been deeply studied in the literature since the early 1990s, and it is beyond our scopes to present here an exhaustive list of references; in the context of non-singular ODEs we highlight the papers [7,8,13,17-20,2830], where the techniques exploited are somehow similar to ours (which shall be described later on).

It is also worth observing that, in addition to ODEs associated with $\Phi$-Laplace operators (which are assumed to be homeomorphisms from $\mathbb{R}$ onto itself), several generalizations of (3) have been proposed and studied, for example, ODEs involving singular $\Phi$-Laplace operators (i.e., with bounded domain) or non-surjective $\Phi$-Laplace operators; see, e.g., $[1,9,16]$.

As regards the existence of heteroclinic solutions of ODEs of the form (3) (i.e., solutions of the BVP (1) with $v_{1} \neq v_{2}$ ), the literature is quite less extended; we highlight the papers $[11,12]$, where the solvability of the BVP

$$
\left\{\begin{array}{l}
\left(a(x(t)) \Phi\left(x^{\prime}(t)\right)\right)^{\prime}=f\left(t, x(t), x^{\prime}(t)\right) \quad \text { a.e. on } \mathbb{R}, \\
x(-\infty)=v_{1}, \quad x(\infty)=v_{2},
\end{array}\right.
$$

is discussed under the assumption that $a$ is strictly positive on $\mathbb{R} \times\left[v_{1}, v_{2}\right]$. We also mention the papers [24,25], where the existence results previously obtained for BVPs of the form (4) are extended to the following problems: 


$$
\left\{\begin{array}{l}
\left(a(t, x(t)) \Phi\left(x^{\prime}(t)\right)\right)^{\prime}=f\left(t, x(t), x^{\prime}(t)\right) \quad \text { a.e. on } \mathbb{R} \\
x(-\infty)=v_{1}, \quad x(\infty)=v_{2}
\end{array}\right.
$$

(again assuming that $a$ is strictly positive). If we further restrict our attention to the problem of the existence of heteroclinic solutions for singular ODEs of the form (3), to the best of our knowledge there are very few results, and just for restricted classes of nonlinearities $f$ (see, e.g., [22,23]).

We now turn to briefly describe our main result and the techniques adopted in order to prove it. As already anticipated, in Theorem 1 we obtain the existence of (at least) one heteroclinic solution of the ODE (3) in a possibly singular setting, that is, we allow the function $a$ to vanish on some set of zero Lebesgue measure. As for the homeomorphism $\Phi$, we just assume that it is strictly increasing and that, roughly put, $\Phi(z) \rightarrow 0$ as $z \rightarrow 0$ faster than some positive power of $z$ (see, precisely, assumption (A1) in Sect. 2). Regarding the function $f$, apart from a Caratheódory regularity assumption, we require a suitable form of the Nagumo-Wintner condition and a "balance" between the behavior of $f(t, x, \cdot)$ with respect to $\Phi$ as $y \rightarrow 0$ and of $f(\cdot, x, y)$ as $t \rightarrow \infty$ (see, precisely, estimate (11) in assumption (B3) and estimate (13) in assumption (B4)).

As is well known, the Nagumo-Wintner condition is a key tool in proving existence results for ODEs (see, e.g., the recent papers $[2,10,14,27]$ ); in our setting, such a condition allows to obtain a priori estimate for the derivative of any solution of (11) on compact intervals (see, precisely, statement (iii) in Proposition 2).

Under the assumptions roughly described above, the proof of our Theorem 1 follows by a suitable combination of a fixed-point technique and of the method of lower/upper solutions. By means of this approach (which has been proved to be very versatile in ODEs Theory, see $[6,26]$ and the very recent papers $[2,10]$ ), we first prove the existence of (at least) one solution $x_{n}$ of the Dirichlet problem

$$
\left\{\begin{array}{l}
\left(\Phi\left(a(t, x(t)) x^{\prime}(t)\right)\right)^{\prime}=f\left(t, x(t), x^{\prime}(t)\right) \text { a.e. on }[0, n] \\
x(0)=\alpha(0), \quad x(n)=\beta(n),
\end{array}\right.
$$

where $n \in \mathbb{N}$ is sufficiently large and $\alpha, \beta$ are, respectively, a lower solution and an upper solution of the ODE (3) with $\alpha \leq \beta$ (see Definition 2 in the next section); then, by crucially exploiting the assumption on the behavior of $f(\cdot, x, y)$ as $t \rightarrow \infty$, we are able to perform a limit argument on the sequence $\left\{x_{n}\right\}_{n}$ to obtain the existence of a solution $x_{0}$ for the BVP in (1) (provided that $\alpha(0)=v_{1}$ and $\beta(n) \rightarrow v_{2}$ ).

It is interesting to observe that the regularity of the solution $x_{0}$ is actually determined by the "problem itself"; in fact, if we suppose that

$$
\mathbf{a}_{\star}(t):=\min _{x \in[\alpha(t), \beta(t)]} a(t, x),
$$

is such that $1 / \mathbf{a}_{\star} \in L_{\text {loc }}^{p}(\Lambda)$ for some $p>1$ (see assumption (B2) in the statement of Theorem 1), then the solution $x_{0}$ turns out to belong to the Sobolev space $W_{\text {loc }}^{1, p}(\Lambda)$. This phenomenon also happens in [2,10], where ODEs of the form (3) are considered on compact intervals (with various types of boundary conditions).

It is worth pointing out that, in the particular case when the function $a$ does not depend on the variable $x$, that is,

$$
a(t, x)=k(t),
$$


for some nonnegative $k \in C(\Lambda, \mathbb{R})$ with $1 / k \in L_{\text {loc }}^{p}(\Lambda)$, then the existence of heteroclinic solutions of (3) is proved in [3]; however, since the case of a function $a$ independent on $x$ is perfectly compatible with our hypotheses, it turns out that Theorem 1 generalizes [3, Theorem 2.4] to a wider class of BVPs.

Just about to illustrate the applicability of our Theorem 1, we shall present in Sect. 5 a quite wide class of examples to which such a theorem does apply. To be more precise, we shall prove that, if the function $a$ takes one of the forms

(*) $a(t, x)=k_{1}(t) k_{2}(x)$ (with $k_{1} \geq 0$ and $\min _{\left[v_{1}, v_{2}\right]} k_{2}>0$ );

(*) $a(t, x)=k_{1}(t)+k_{2}(x)\left(\right.$ with $\left.k_{1}, k_{2} \geq 0\right)$

and if $f$ can be written as

$$
f(t, x, y)=f_{1}(t, x) f_{2}(y),
$$

(with $f_{2}(0)=0$ and $f_{1}, f_{2}$ satisfying suitable growth assumptions), then our Theorem 1 applies (whatever the chosen $v_{1}, v_{2} \in \mathbb{R}$ ). This is the case, e.g., of

$$
\left\{\begin{array}{l}
\left(\sinh \left(k(t) e^{x(t)} x^{\prime}(t)\right)\right)^{\prime}=-\frac{\left|x^{\prime}(t)\right|}{\varrho+\cos ^{2}(t)} \text { a.e. on } \Lambda, \\
x(0)=0, \quad x(\infty)=1,
\end{array}\right.
$$

where $\varrho>0$ and $k$ is the unique 1-periodic prolongation of

$$
k_{0}(t)=\sqrt{t(1-t)} \quad(\text { with } 0 \leq t \leq 1) .
$$

We notice that, in the above example, the $\Phi$-Laplace operator $\Phi(z)=\sinh (z)$ is not homogeneous and it grows at infinity faster than any power of $z$.

Even if the assumptions of Theorem 1 are general enough to be satisfied by several examples of BVPs, it should be of interest to discuss whether these assumptions are sharp or not; we plain to return on this topic in a forthcoming paper.

Plan of the paper: A short plan of the paper is now in order.

- In Sect. 2, we introduce the standing assumptions used throughout the paper and we state our main result, namely Theorem 1.

- In Sect. 3, we establish some preliminary results concerning the solvability of (1) on $I_{n}=[0, n]$ (with $n \in \mathbb{N}$ sufficiently large).

- In Sect. 4, we prove Theorem 1 by a limit argument.

- In Sect. 5, we present a wide class of BVPs to which Theorem 1 applies.

\section{General assumptions and main results}

Let $\Lambda:=[0, \infty)$ and let $v_{1}, v_{2} \in \mathbb{R}$. As we already discussed in "Introduction," the present paper aims to study the solvability of strongly nonlinear and non-autonomous boundary value problems of the following type

$$
\left\{\begin{array}{l}
\left(\Phi\left(a(t, x(t)) x^{\prime}(t)\right)\right)^{\prime}=f\left(t, x(t), x^{\prime}(t)\right) \text { a.e. on } \Lambda \\
x(0)=v_{1}, \quad x(\infty)=v_{2} .
\end{array}\right.
$$

As in [2,3], we require $\Phi, a$ and $f$ to satisfy the following assumptions: 
(A1) $\Phi: \mathbb{R} \rightarrow \mathbb{R}$ is a strictly increasing homeomorphism such that $\Phi(0)=0$ and

$$
\liminf _{z \rightarrow 0} \frac{\Phi(z)}{z^{\rho}}>0 \text { for a suitable } \rho>0 ;
$$

(A2) $a: \Lambda \times \mathbb{R} \rightarrow \mathbb{R}$ is nonnegative and continuous on $\Lambda \times \mathbb{R}$;

(A3) $f: \Lambda \times \mathbb{R}^{2} \rightarrow \mathbb{R}$ is a Caratheódory function, that is,

$(\star) t \mapsto f(t, x, y)$ is measurable on $\Lambda$ for every fixed $(x, y) \in \mathbb{R}^{2}$;

$(\star)(x, y) \mapsto f(t, x, y)$ is continuous for a.e. $t \in \Lambda$.

We now specify what we mean by a solution of problem (5).

Definition 1 We say that a continuous function $x: \Lambda \rightarrow \mathbb{R}$ is a solution of the boundary value problem (1) if it satisfies the following properties:

(1) $x \in W_{\mathrm{loc}}^{1,1}(\Lambda)$ and $t \mapsto \Phi\left(a(t, x(t)) x^{\prime}(t)\right) \in W_{\mathrm{loc}}^{1,1}(\Lambda)$;

(2) $\left(\Phi\left(a(t, x(t)) x^{\prime}(t)\right)\right)^{\prime}=f\left(t, x(t), x^{\prime}(t)\right)$ for almost every $t \in \Lambda$;

(3) $x(0)=v_{1}$ and $x(t) \rightarrow v_{2}$ as $t \rightarrow \infty$.

If $x$ fulfills only (1) and (2), we say that $x$ is a solution of the ODE

$$
\left(\Phi\left(a(t, x(t)) x^{\prime}(t)\right)\right)^{\prime}=f\left(t, x(t), x^{\prime}(t)\right) .
$$

As for the case of the non-singular boundary value problems studied in [11] and in [12], our approach to the solvability of (5) is based on the so-called method of lower/upper solutions, according to the following definition.

Definition 2 We say that a continuous function $\alpha: \Lambda \rightarrow \mathbb{R}$ is a lower [resp. upper] solution of the ODE (7) if it satisfies the following properties:

(1) $\alpha \in W_{\mathrm{loc}}^{1,1}(\Lambda)$ and $t \mapsto \Phi\left(a(t, \alpha(t)) \alpha^{\prime}(t)\right) \in W_{\mathrm{loc}}^{1,1}(\Lambda)$;

(2) $\left(\Phi\left(a(t, \alpha(t)) \alpha^{\prime}(t)\right)\right)^{\prime} \geq[\leq] f\left(t, \alpha(t), \alpha^{\prime}(t)\right)$ for almost every $t \in \Lambda$.

Remark 1 If $x \in W_{\mathrm{loc}}^{1,1}(\Lambda)$ is any function such that

$$
t \mapsto \Phi\left(a(t, x(t)) x^{\prime}(t)\right) \in W_{\mathrm{loc}}^{1,1}(\Lambda)
$$

(as for the case of lower/upper solutions of the ODE (7)), from the fact that $\Phi$ is a homeomorphism we infer the existence of a unique $\mathcal{A}_{x} \in C(\Lambda, \mathbb{R})$ such that

$$
\mathcal{A}_{x}(t)=a(t, x(t)) x^{\prime}(t) \quad \text { for a.e. } t \in \Lambda .
$$

We are ready to state the main existence result of this paper.

Theorem 1 Let us suppose that, together with the "structural" assumptions (A1)-(A3), the following additional hypotheses are satisfied:

(B1) There exists a pair of lower and upper solutions $\alpha, \beta \in W_{\mathrm{loc}}^{1,1}(\Lambda)$ of the ODE (7) such that $\alpha \leq \beta$ on $\Lambda$; moreover, there exists $T_{0}>0$ such that

$$
\beta \text { is increasing on }\left[T_{0}, \infty\right) \quad \text { and } \quad \lim _{t \rightarrow \infty} \beta(t)=: \beta_{0} \in \mathbb{R} \text {. }
$$


(B2) There exists a real $p>1$ such that, setting

$$
\mathbf{a}_{\star}(t):=\min _{x \in[\alpha(t), \beta(t)]} a(t, x) \geq 0,
$$

it holds that $1 / \mathbf{a}_{\star} \in L_{\mathrm{loc}}^{p}(\Lambda)$;

(B3) There exist a constant $H>0$, a nonnegative function $\mu \in L^{q}\left(\left[0, T_{0}\right]\right.$ ) (for some $1<q \leq \infty)$, a nonnegative function $l \in L^{1}\left(\left[0, T_{0}\right]\right)$ and a nonnegative measurable function $\psi:(0, \infty) \rightarrow(0, \infty)$ such that

$$
\begin{aligned}
& \text { ( }) 1 / \psi \in L_{\mathrm{loc}}^{1}(0, \infty) \text { and } \int_{1}^{\infty} \frac{1}{\psi(t)} \mathrm{d} t=\infty \text {; } \\
& (\star)|f(t, x, y)| \leq \psi(|\Phi(a(t, x) y)|) \cdot\left(l(t)+\mu(t)|y|^{\frac{q-1}{q}}\right) ;
\end{aligned}
$$

for a.e. $t \in\left[0, T_{0}\right]$, every $x \in[\alpha(t), \beta(t)]$ and any $y \in \mathbb{R}$ with $|a(t, x) y| \geq H ;$

(B4) There exists a real constant $\theta>1$ with the following property: For every fixed $L>0$ it is possible to find a nonnegative function $\eta_{L} \in L^{1}(\Lambda)$ and a continuous function $K_{L} \in W_{\text {loc }}^{1,1}(\Lambda)$, null on $\left[0, T_{0}\right]$ and strictly increasing on the whole of $\left[T_{0}, \infty\right)$, satisfying the following properties:

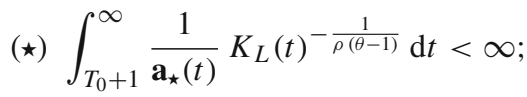

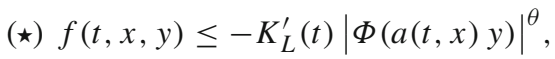
for a.e. $t \geq T_{0}$, every $x \in[\alpha(t), \beta(t)]$ and every $y \in \mathbb{R}$ with $|y| \leq \mathcal{N}_{L}(t) / a(t, x)$;

$(\star)-\eta_{L}(t) \leq f(t, x, y) \leq \eta_{L}(t)$, for a.e. $t \in \Lambda$, every $x \in[\alpha(t), \beta(t)]$ and every $y \in \mathbb{R}$ with $|y| \leq\left(\mathcal{N}_{L}(t) / \mathbf{a}_{\star}(t)\right)+\left|\alpha^{\prime}(t)\right|+\left|\beta^{\prime}(t)\right| ;$

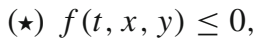

for a.e. $t \geq T_{0}$, every $x \in[\alpha(t), \beta(t)]$ and every $y \in \mathbb{R}$ with $|y| \leq\left(\mathcal{N}_{L}(t) / \mathbf{a}_{\star}(t)\right)+\left|\alpha^{\prime}(t)\right|+\left|\beta^{\prime}(t)\right|$.

Here, we have used the notation

$$
\mathcal{N}_{L}(t):=\Phi^{-1}\left\{\left(\Phi(L)^{1-\theta}+(\theta-1) K_{L}(t)\right)^{1 /(1-\theta)}\right\}
$$

Then, there exists (at least) one solution $x \in W_{\mathrm{loc}}^{1, p}(\Lambda)$ of the problem

$$
\begin{cases}\left(\Phi\left(a(t, x(t)) x^{\prime}(t)\right)\right)^{\prime}=f\left(t, x(t), x^{\prime}(t)\right) & \text { for a.e. } t \in \Lambda, \\ \alpha(t) \leq x(t) \leq \beta(t) & \text { for any } t \in \Lambda, \\ x(0)=\alpha(0), \quad x(\infty)=\beta_{0} . & \end{cases}
$$

Here, $p>1$ is the same exponent appearing in assumption (B2).

Remark 2 Some remarks on the assumptions of Theorem 1 are in order.

1. The existence of lower/upper solutions for the ODE (7) is not obvious, and in general, it could be a very delicate issue; we highlight the papers $[6,26]$ (and the references therein) for some results concerning this topics. 
2. In the particular case, when

$$
f(t, x, 0)=0 \quad \text { for all } t \in \Lambda \text { and } x \in \mathbb{R},
$$

it is very easy to see that any constant function is a solution of the ODE (7), so that it is both a lower solution and an upper solution.

3. When the lower and the upper solution $\alpha, \beta$ in assumption (B1) can be chosen to be constant on $\Lambda$, the estimate (14) and the sign condition (15) in assumption (B4) can be rewritten, respectively, as

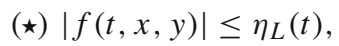

$$
\begin{aligned}
& \text { for a.e. } t \in \Lambda \text {, every } x \in[\alpha, \beta] \text { and every } y \in \mathbb{R} \\
& \text { with }|y| \leq\left(\mathcal{N}_{L}(t) / \mathbf{a}_{\star}(t)\right) \text {; }
\end{aligned}
$$

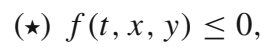

$$
\begin{aligned}
& \text { for a.e. } t \geq T_{0} \text {, every } x \in[\alpha, \beta] \text { and every } y \in \mathbb{R} \\
& \text { with }|y| \leq\left(\mathcal{N}_{L}(t) / \mathbf{a}_{\star}(t)\right) \text {. }
\end{aligned}
$$

Remark 3 For a future reference, we collect in the present remark a couple of consequences of assumption (B1) we shall frequently use throughout the paper.

(i) Since $1 / \mathbf{a}_{\star} \in L_{\text {loc }}^{p}(\Lambda)$ for some $p>1$, there exists a set $E \subseteq \Lambda$ with vanishing Lebesgue measure such that $\mathbf{a}_{\star}>0$ on $\Lambda \backslash E$; as a consequence,

$$
a(t, x) \geq \mathbf{a}_{\star}(t)>0 \text { for every }(t, x) \in(\Lambda \backslash E) \times \mathbb{R} .
$$

(ii) If $E$ is as in (i), by (18) and the very definition of $\mathbf{a}_{\star}$ we have

$$
0<\frac{1}{a(t, x)} \leq \frac{1}{\mathbf{a}_{\star}(t)}, \quad \text { for a.e. }(t, x) \in(\Lambda \backslash E) \times \mathbb{R} .
$$

Before embarking on the proof of Theorem 1 (which is quite long and technical), we briefly describe the main steps on which such a proof is based.

(I) First of all, given any natural $n>T_{0}$, we shall show that there exists at least one solution $u_{n}$ on $I_{n}:=[0, n]$ of the boundary value problem

$$
\left(P_{n}\right) \quad\left\{\begin{array}{l}
\left(\Phi\left(a(t, x(t)) x^{\prime}(t)\right)\right)^{\prime}=f\left(t, x(t), x^{\prime}(t)\right) \text { for a.e. } t \in I_{n}, \\
x(0)=\alpha(0), \quad x(n)=\beta(n) .
\end{array}\right.
$$

To this aim, we shall exploit some existence results established in [2].

(II) Once Step I has been completed, we shall prove that the sequence $\left\{u_{n}\right\}_{n}$ converges (up to a subsequence) to a function $x_{0} \in W^{1, p}(\Lambda)$ which is a heteroclinic solution of (7), that is, $x_{0}$ is a solution of (5).

Plan of the paper: Bearing in mind the scheme for the proof of Theorem 1 just described, we organize the paper in the following way:

- in Sect. 3, we shall prove the solvability of problem $\left(P_{n}\right)$ (for every $n>T_{0}$ ) and we shall show some useful features of its solutions;

- in Sect. 4, we shall use a convergence argument in order to prove the solvability of problem (17), as described in Step II. 


\section{Existence of solutions on $[0, n]$}

Throughout this section, we take for fixed all the notations introduced so far; furthermore, we assume that all the hypotheses in Theorem 1 are satisfied.

Let $n \in \mathbb{N}, n>T_{0}$ be arbitrary but fixed. As anticipated, our main aim is to prove the existence of at least one solution $u_{n}$ of the following problem

$$
\left(P_{n}\right) \quad\left\{\begin{array}{l}
\left(\Phi\left(a(t, x(t)) x^{\prime}(t)\right)\right)^{\prime}=f\left(t, x(t), x^{\prime}(t)\right) \text { for a.e. } t \in I_{n}=[0, n], \\
x(0)=\alpha(0), \quad x(n)=\beta(n) .
\end{array}\right.
$$

To this end, we follow an approach similar to that exploited in [11] but adapted to our singular setting, in which the function $a$ can vanish (see also [2]).

First of all, let $J:=\left[0, T_{0}\right]$ (where $T_{0}$ is an assumption (B1)) and let

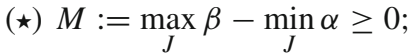

$$
\begin{aligned}
& (\star) a_{0}:=\max \{a(t, x): t \in J \text { and } \alpha(t) \leq x \leq \beta(t)\} \text {. }
\end{aligned}
$$

Moreover, if $H>0$ is as in assumption (B3), we fix a real $N>0$ such that

$$
N>\max \left\{\frac{M a_{0}}{T_{0}}, H\right\},
$$

and, according to this choice of $N$, we let $L>N$ be such that (see (10))

$$
\min \left\{\int_{\Phi(N)}^{\Phi(L)} \frac{\mathrm{d} s}{\psi(s)}, \int_{-\Phi(-N)}^{-\Phi(-L)} \frac{\mathrm{d} s}{\psi(s)}\right\}>\|l\|_{L^{1}(J)}+\|\mu\|_{L^{q}(J)}(M)^{\frac{q-1}{q}},
$$

With reference to assumption (B4), we finally set

$$
\gamma_{0}:=\mathcal{N}_{L} / \mathbf{a}_{\star}
$$

Remark 4 We explicitly observe, for a future reference, that the function $\gamma_{0}$ defined in (23) satisfies the following integrability property:

$$
\gamma_{0} \in L_{\mathrm{loc}}^{p}(\Lambda) \cap L^{1}(\Lambda) .
$$

In fact, since $\mathcal{N}_{L}$ is continuous on $\Lambda$ (as is very easy to see) and $1 / \mathbf{a}_{\star} \in L_{\text {loc }}^{p}(\Lambda)$, we immediately derive that $\gamma_{0} \in L_{\mathrm{loc}}^{p}(\Lambda)$. To prove that $\gamma_{0}$ also belongs to the space $L^{1}(\Lambda)$ we first notice that, by condition (6) in assumption (A1), we have

$$
\limsup _{\xi \rightarrow 0^{+}} \frac{\Phi^{-1}(\xi)}{\xi^{1 / \rho}}=\lambda<\infty
$$

as a consequence, since $\left(\Phi(L)^{1-\theta}+(\theta-1) K_{L}\right)^{1 /(1-\theta)}$ is nonnegative and bounded from above by $\Phi(L)$ on $\Lambda$, we obtain (here, $T>T_{0}$ is arbitrarily fixed)

$$
\int_{0}^{\infty} \frac{\mathcal{N}_{L}(t)}{\mathbf{a}_{\star}(t)} \mathrm{d} t \leq \mathbf{c}\left(\int_{0}^{T} \frac{1}{\mathbf{a}_{\star}(t)} \mathrm{d} t+\int_{T}^{\infty} \frac{1}{\mathbf{a}_{\star}(t)} K_{L}(t)^{-\frac{1}{\rho(\theta-1)}} \mathrm{d} t\right) .
$$

Owing to (12) in assumption (B4) and again to the fact that $1 / \mathbf{a}_{\star} \in L_{\text {loc }}^{p}(\Lambda)$, we conclude that $\gamma_{0}$ is in $L^{1}(\Lambda)$, as claimed. 
We now consider the truncating operators $\mathcal{T}, \mathcal{D}$ defined, respectively, as follows:

$$
\begin{aligned}
\mathcal{T}: W^{1, p}\left(I_{n}\right) \longrightarrow W^{1,1}\left(I_{n}\right), & \mathcal{T}(x)(t):= \begin{cases}\alpha(t), & \text { if } x(t)<\alpha(t) ; \\
x(t), & \text { if } x(t) \in[\alpha(t), \beta(t)] ; \\
\beta(t), & \text { if } x(t)>\beta(t) ;\end{cases} \\
\mathcal{D}: L^{1}\left(I_{n}\right) \longrightarrow L^{1}\left(I_{n}\right), & \mathcal{D}(z)(t):= \begin{cases}-\gamma_{0}(t), & \text { if } z(t)<-\gamma_{0}(t) ; \\
z(t), & \text { if }|z(t)| \leq \gamma_{0}(t) ; \\
\gamma_{0}(t), & \text { if } z(t)>\gamma_{0}(t) .\end{cases}
\end{aligned}
$$

We also introduce the truncated function $f^{*}: I_{n} \times \mathbb{R}^{2} \rightarrow \mathbb{R}$ defined by

$$
f^{*}(t, x, y):= \begin{cases}f\left(t, \beta(t), \beta^{\prime}(t)\right)+\arctan (x-\beta(t)), & \text { if } x>\beta(t) ; \\ f(t, x, y), & \text { if } x \in[\alpha(t), \beta(t)] ; \\ f\left(t, \alpha(t), \alpha^{\prime}(t)\right)+\arctan (x-\alpha(t)), & \text { if } x<\alpha(t) .\end{cases}
$$

Remark 5 As observed in the very recent paper [3], the two terms

$$
\arctan (x-\alpha(t)) \text { and } \arctan (x-\beta(t)),
$$

imply the following relations among $f$ and $f^{*}$, valid for any $(t, x, y) \in I_{n} \times \mathbb{R}^{2}$ :

$-f^{*}(t, x, y)<f\left(t, \alpha(t), \alpha^{\prime}(t)\right)$ if $x<\alpha(t)$;

- $f^{*}(t, x, y)>f\left(t, \beta(t), \beta^{\prime}(t)\right)$ if $x>\beta(t)$.

These relations are crucial in the proof of Proposition 2.

By means of the function $f^{*}$ and of the operators $\mathcal{T}$ and $\mathcal{D}$ just defined, we can introduce the following truncated version of our problem $\left(P_{n}\right)$ :

$$
\left\{\begin{array}{l}
\left(\Phi\left(a(t, \mathcal{T}(x)(t)) x^{\prime}(t)\right)\right)^{\prime}=f^{*}\left(t, x(t), \mathcal{D}\left(\mathcal{T}(x)^{\prime}(t)\right)\right), \quad \text { a.e. on } I_{n}, \\
x(0)=\alpha(0), \quad x(n)=\beta(n) .
\end{array}\right.
$$

Our aim is to show that problem (24) has (at least) one solution $u_{n} \in W^{1, p}\left(I_{n}\right)$. To this end, we use the following abstract existence result established in [2].

Theorem 2 ([2, Theorem 2.5]) Let $A: W^{1, p}\left(I_{n}\right) \subseteq C\left(I_{n}, \mathbb{R}\right) \rightarrow C\left(I_{n}, \mathbb{R}\right)$ and $F$ : $W^{1, p}\left(I_{n}\right) \rightarrow L^{1}\left(I_{n}\right)$ be general operators satisfying the following properties:

(H1) A is continuous with respect to the uniform topology of $C\left(I_{n}, \mathbb{R}\right)$; moreover, there exist two functions $h_{1}, h_{2} \in C\left(I_{n}, \mathbb{R}\right)$ such that

(H1) $h_{1}, h_{2} \geq 0$ on $I$ and $1 / h_{1}, 1 / h_{2} \in L^{p}\left(I_{n}\right)$;

$(\mathrm{H} 1)_{2} h_{1}(t) \leq A(x)(t) \leq h_{2}(t)$ for every $x \in W^{1, p}\left(I_{n}\right)$ and every $t \in I_{n}$;

(H2) $F$ is continuous (with respect to the usual norms) and there exists a nonnegative function $\Theta \in L^{1}\left(I_{n}\right)$ such that

$$
|F(x)(t)| \leq \Theta(t) \text { for every } x \in W^{1, p}\left(I_{n}\right) \text { and a.e. } t \in I_{n} .
$$

Then, for every $v_{1}, v_{2} \in \mathbb{R}$ there exists a solution $x \in W^{1, p}\left(I_{n}\right)$ of the problem

$$
\left\{\begin{array}{l}
\left(\Phi\left(A(x)(t) x^{\prime}(t)\right)\right)^{\prime}=F(x)(t) \quad \text { a.e. on } I_{n}, \\
x(0)=v_{1}, \quad x(n)=v_{2} .
\end{array}\right.
$$


More precisely, there exists a function $x \in W^{1, p}\left(I_{n}\right)$ such that

(a) $\Phi \circ\left(A(x) \cdot x^{\prime}\right) \in W^{1,1}\left(I_{n}\right)$ and $\left(\Phi \circ\left(A(x) \cdot x^{\prime}\right)\right)^{\prime}=F$ in $L^{1}\left(I_{n}\right)$;

(b) $x(0)=v_{1}$ and $x(n)=v_{2}$.

Thanks to Theorem 2, we can prove the existence of a solution of (24).

Proposition 1 Let the above assumptions and notation apply. Then, there exists (at least) one solution $u_{n} \in W^{1, p}\left(I_{n}\right)$ of the Dirichlet problem (24).

Proof On account of Theorem 2, we consider the following operators:

$$
\begin{aligned}
& A: W^{1, p}\left(I_{n}\right) \longrightarrow C\left(I_{n}, \mathbb{R}\right), \quad A(x)(t):=a(t, \mathcal{T}(x)(t)), \\
& F: W^{1, p}\left(I_{n}\right) \longrightarrow L^{1}\left(I_{n}\right), \quad F(x)(t):=f^{*}\left(t, x(t), \mathcal{D}\left(\mathcal{T}(x)^{\prime}\right)(t)\right) .
\end{aligned}
$$

By means of these operators (and setting $v_{1}:=\alpha(0), v_{2}:=\beta(n)$ ), we can rewrite problem (24) in the following abstract form:

$$
\left\{\begin{array}{l}
\left(\Phi\left(A(x)(t) x^{\prime}(t)\right)\right)^{\prime}=F(x)(t), \quad \text { a.e. on } I_{n}, \\
x(0)=v_{1}, \quad x(n)=v_{2} .
\end{array}\right.
$$

We claim that $A$ and $F$ satisfy assumptions (H1) and (H2) in Theorem 2.

As for the operator $A$ we first notice that, since $\mathcal{T}$ is continuous as an operator on $C\left(I_{n}, \mathbb{R}\right)$ (as is very easy to see) and since, by definition,

$$
\alpha_{n}:=\min _{I_{n}} \alpha \leq \alpha(t) \leq \mathcal{T}(x)(t) \leq \beta(t) \leq \max _{I_{n}} \beta=: \beta_{n} \quad \text { for every } t \in I_{n},
$$

the uniform continuity of $a$ on $I_{n} \times\left[\alpha_{n}, \beta_{n}\right]$ implies that $A$ is continuous with respect to the uniform topology of $C\left(I_{n}, \mathbb{R}\right)$. Moreover, if $x \in W^{1, p}\left(I_{n}\right)$, one has

$$
A(x)(t)=a(t, \mathcal{T}(x)(t)) \leq \max _{I_{n} \times\left[\alpha_{n}, \beta_{n}\right]} a=: a_{n} \text { for every } t \in I_{n} .
$$

Finally, if $\mathbf{a}_{\star}$ is as in (9), from assumption (B2) we derive that $\mathbf{a}_{\star} \geq 0$ on $I_{n}$ and that $1 / \mathbf{a}_{\star} \in L^{p}\left(I_{n}\right)$; furthermore, by the very definition of $\mathbf{a}_{\star}$ and $\mathcal{T}$ we get

$$
A(x)(t) \geq \min _{\xi \in[\alpha(t), \beta(t)]} a(t, \xi)=\mathbf{a}_{\star}(t) \quad\left(x \in W^{1, p}\left(I_{n}\right) \text { and } t \in I_{n}\right),
$$

and thus $A$ satisfies assumption (H1) with the choice $h_{1}=\mathbf{a}_{\star}$ and $h_{2} \equiv a_{n}>0$.

As for the operator $F$, by arguing exactly as in [11, Theorem 3.1] (where the same truncated function is considered), we see that $F$ is continuous and that

$$
\begin{aligned}
|F(x)(t)| \leq & \max \left\{\eta_{L}(t),\left|f\left(t, \alpha(t), \alpha^{\prime}(t)\right)\right|,\left|f\left(t, \beta(t), \beta^{\prime}(t)\right)\right|\right\}+\frac{\pi}{2} \\
& (\text { by crucially using }(14)) \\
& \leq \eta_{L}(t)+\frac{\pi}{2}=: \Theta(t),
\end{aligned}
$$

for every $x \in W^{1, p}\left(I_{n}\right)$ and a.e. $t \in I_{n}$. (Here, $\eta_{L} \in L^{1}(\Lambda)$ is the function appearing in assumption (B4) and associated with the constant $L$ chosen in (22).) Since, obviously, $\Theta \in L^{1}\left(I_{n}\right)$, we conclude that $F$ satisfies (H2) in Theorem 2 .

We are then entitled to apply Theorem 2, which ensures the existence of (at least) one solution $u_{n} \in W^{1, p}\left(I_{n}\right)$ of the truncated problem (24). 
Remark 6 It is worth noting that, if $x \in W^{1, p}\left(I_{n}\right)$ is an arbitrary solution of the truncated problem (24), owing to (a) in the statement of Theorem 2 (and to the very definition of the operator $A$, see (25)) we have

$$
t \mapsto \Phi\left(A(x)(t) \cdot x^{\prime}(t)\right)=\Phi\left(a(t, \mathcal{T}(x)(t)) x^{\prime}(t)\right) \in W^{1,1}\left(I_{n}\right) ;
$$

as a consequence, by arguing exactly as in Remark 1 (and following the same notation), there exists a unique continuous function $\mathcal{A}_{x}$ on $I_{n}$ such that

$$
\mathcal{A}_{x}(t)=a(t, \mathcal{T}(x)(t)) x^{\prime}(t)=A(x)(t) \text { for a.e. } t \in I_{n} .
$$

Now we have established the solvability of problem (24), we proceed by showing that any solution of this problem is actually a solution of $\left(P_{n}\right)$.

To begin with, we state the following useful proposition.

Proposition 2 With the above assumptions and notation, let $u_{n} \in W^{1, p}\left(I_{n}\right)$ be any solution of the truncated problem (24). Then, the following facts hold:

(i) $\alpha(t) \leq u_{n}(t) \leq \beta(t)$ for every $t \in I_{n}$, whence

$$
\begin{aligned}
& \text { (*) } \mathcal{T}\left(u_{n}\right) \equiv u_{n} \text { on } I_{n} ; \\
& \text { (*) } \mathcal{A}_{u_{n}}(t)=a\left(t, u_{n}(t)\right) u_{n}^{\prime}(t) \text { for a.e. } t \in I_{n} ; \\
& \text { (*) } \mathcal{D}\left(\mathcal{T}\left(u_{n}\right)^{\prime}\right)=u_{n}^{\prime} \text { a.e. on } I_{n} .
\end{aligned}
$$

(ii) $\left|\mathcal{A}_{u_{n}}(t)\right|<L$ for every $t \in J=\left[0, T_{0}\right]$;

(iii) $\left|u_{n}^{\prime}(t)\right|<L / \mathbf{a}_{\star}(t)$ for a.e. $t \in J$.

Proof (i) The proof of this statement rests on some ideas which are similar to that exploited in the demonstration of [10, Theorem 3.1 (Claim II)]; here, we limit ourselves to point out that the two terms

$$
\arctan (x-\alpha(t)) \text { and } \arctan (x-\beta(t)),
$$

in the definition of $f^{*}$ play a crucial rôle, and we refer to [2] for all the details.

(ii) Also the proof if this statement exploits some ideas which are similar to that contained in [10]; however, we present all the details since the modified version of the Nagumo-Wintner condition in assumption (B3) intervenes here.

To begin with, if $N$ is as in (21), we claim that

$$
\min _{t \in J}\left|\mathcal{A}_{u_{n}}(t)\right| \leq N
$$

To prove (29), we argue by contradiction, and to fix ideas, we assume that

$$
\mathcal{A}_{u_{n}}(t)=a\left(t, u_{n}(t)\right) u_{n}^{\prime}(t)>N \text { for a.e. } t \in J
$$

hence, in particular, $u_{n}^{\prime}(t)>0$ for almost every $t \in J$ (see assumption (B2)). If we now integrate both sides of this inequality on $J=\left[0, T_{0}\right]$, we get

$$
\int_{0}^{T_{0}} \mathcal{A}_{u_{n}}(t) \mathrm{d} t=\int_{0}^{T_{0}} a\left(t, u_{n}(t)\right) u_{n}^{\prime}(t) \mathrm{d} t>N T_{0} ;
$$

as a consequence, by statement (i), (19), (20) and the choice of $N$ one has

$$
N T_{0}<\int_{0}^{T_{0}} a\left(t, u_{n}(t)\right) u_{n}^{\prime}(t) \mathrm{d} t \leq a_{0} \cdot \int_{0}^{T_{0}} u_{n}^{\prime}(t) \mathrm{d} t
$$




$$
\begin{aligned}
& \leq a_{0} \cdot\left(u_{n}\left(T_{0}\right)-u_{n}(0)\right) \leq a_{0} \cdot\left(\beta\left(T_{0}\right)-\alpha(0)\right) \\
& \leq a_{0} M<N T_{0} .
\end{aligned}
$$

This is clearly a contradiction, so that $\mathcal{A}_{u_{n}} \leq N$ on $J$. Arguing analogously, one can prove that $\mathcal{A}_{u_{n}} \geq-N$ on $J$, whence (29) holds true.

With (29) at hand, we now prove statement (ii). Arguing once again by contradiction, we assume the existence of a point $\bar{t} \in J$ such that

$$
\left|\mathcal{A}_{u_{n}}(\bar{t})\right| \geq L
$$

moreover, to fix ideas, we suppose that $\mathcal{A}_{u_{n}}(\bar{t}) \geq L$. Since, by the choice of $L$, we have $L>N$, from (29) (and the continuity of $\mathcal{A}_{u_{n}}$ ) we derive the existence of two points $t_{1}, t_{2} \in J$, with (to fix ideas) $t_{1}<t_{2}$, such that

(a) $\mathcal{A}_{u_{n}}\left(t_{1}\right)=N$ and $\mathcal{A}_{u_{n}}\left(t_{2}\right)=L$;

(b) $0<N<\mathcal{A}_{u_{n}}(t)<L$ for every $t \in\left(t_{1}, t_{2}\right) \subseteq J$;

in particular, reminding that $\Phi$ is increasing and $\Phi(0)=0$ we get

$$
\Phi\left(\mathcal{A}_{u_{n}}(t)\right)>\Phi(0)=0 \text { for every } t \in\left(t_{1}, t_{2}\right) .
$$

Now, since $\left(t_{1}, t_{2}\right) \subseteq J$ and, by definition, $\mathcal{N}_{L} \equiv L$ on $J$ (see (16)), from (b) we infer that (see also (27) and Remark 3)

$$
0<u_{n}^{\prime}(t) \leq \frac{L}{\mathbf{a}_{\star}(t)}=\frac{\mathcal{N}_{L}(t)}{\mathbf{a}_{\star}(t)} \stackrel{(23)}{=} \gamma_{0}(t), \quad \text { for a.e. } t \in\left(t_{1}, t_{2}\right) ;
$$

by combining (31) with the definition of $\mathcal{D}$ and the fact that $u_{n}$ solves (24) on $I_{n}$, we then get [see also (27), (28) and the definition of $f^{*}$ ]

$$
\begin{aligned}
\left(\Phi\left(\mathcal{A}_{u_{n}}(t)\right)\right)^{\prime} & =\left(\Phi\left(a\left(t, u_{n}(t)\right) u_{n}^{\prime}(t)\right)\right)^{\prime}=f^{*}\left(t, u_{n}(t), \mathcal{D}\left(u_{n}^{\prime}\right)(t)\right), \\
& =f\left(t, u_{n}(t), u_{n}^{\prime}(t)\right), \quad \text { for a.e. } t \in\left(t_{1}, t_{2}\right) .
\end{aligned}
$$

On the other hand, since $N>H$ (see (21)), again from (b) we infer that

$$
a\left(t, u_{n}(t)\right) u_{n}^{\prime}(t)=\mathcal{A}_{u_{n}}(t)>H, \quad \text { for a.e. } t \in\left(t_{1}, t_{2}\right) .
$$

Gathering together (33), statement (i) and the trivial fact that $\left(t_{1}, t_{2}\right) \subseteq J$, we are then entitled to apply estimate (11) in assumption (B3), obtaining

$$
\begin{aligned}
\left|\left(\Phi\left(\mathcal{A}_{u_{n}}(t)\right)\right)^{\prime}\right| & \stackrel{(32)}{=}\left|f\left(t, u_{n}(t), u_{n}^{\prime}(t)\right)\right| \\
& \stackrel{(11)}{\leq} \psi\left(\left|\Phi\left(a\left(t, u_{n}(t)\right) u_{n}^{\prime}(t)\right)\right|\right) \cdot\left(l(t)+\mu(t)\left(u_{n}^{\prime}(t)\right)^{\frac{q-1}{q}}\right) \\
& =\psi\left(\left|\Phi\left(\mathcal{A}_{u_{n}}(t)\right)\right|\right) \cdot\left(l(t)+\mu(t)\left(u_{n}^{\prime}(t)\right)^{\frac{q-1}{q}}\right) \\
& \stackrel{(30)}{=} \psi\left(\Phi\left(\mathcal{A}_{u_{n}}(t)\right)\right) \cdot\left(l(t)+\mu(t)\left(u_{n}^{\prime}(t)\right)^{\frac{q-1}{q}}\right) \quad\left(\text { a.e. on }\left(t_{1}, t_{2}\right)\right) .
\end{aligned}
$$

As a consequence, by exploiting this last inequality we obtain

$$
\begin{aligned}
\int_{\Phi(N)}^{\Phi(L)} \frac{1}{\psi(s)} \mathrm{d} s & =\int_{\Phi\left(\mathcal{A}_{u_{n}}\left(t_{1}\right)\right)}^{\Phi\left(\mathcal{A}_{u_{n}}\left(t_{2}\right)\right)} \frac{1}{\psi(s)} \mathrm{d} s \\
& \leq \int_{t_{0}}^{t_{1}} \frac{\left|\left(\Phi\left(\mathcal{A}_{u_{n}}(t)\right)\right)^{\prime}\right|}{\psi\left(\Phi\left(\mathcal{A}_{u_{n}}(t)\right)\right)} \mathrm{d} t \leq \int_{t_{0}}^{t_{1}}\left(l(t)+\mu(t)\left(u_{n}^{\prime}(t)\right)^{\frac{q-1}{q}}\right) \mathrm{d} t
\end{aligned}
$$


(by Hölder's inequality)

$$
\begin{aligned}
& \leq\|l\|_{L^{1}(J)}+\|\mu\|_{L^{q}(J)} \cdot\left(\int_{t_{0}}^{t_{1}} u_{n}^{\prime}(t) \mathrm{d} t\right)^{\frac{q-1}{q}} \\
& \leq\|l\|_{L^{1}(J)}+\|\mu\|_{L^{q}(J)} \cdot\left(u_{n}\left(t_{1}\right)-u_{n}\left(t_{0}\right)\right)^{\frac{q-1}{q}} \\
& \quad\left(\text { since, by }(\mathrm{i}), u_{u}\left(t_{1}\right)-u_{n}\left(t_{0}\right) \leq \beta\left(t_{1}\right)-\alpha\left(t_{0}\right) \leq M\right) \\
& \leq\|l\|_{L^{1}(J)}+\|\mu\|_{L^{q}(J)} \cdot(M)^{\frac{q-1}{q}} .
\end{aligned}
$$

This is in contradiction with the choice of $L$ (see (22)), and thus $\mathcal{A}_{u_{n}} \leq L$ on $J$. Arguing analogously, one can prove that $\mathcal{A}_{u_{n}} \geq-L$ on $J$, and the statement is therefore completely demonstrated.

(iii) It is a direct consequence of (ii) and (27) (see also Remark 3).

The next proposition concerns the behavior of $u_{n}$ on the interval $\left[T_{0}, n\right]$.

Proposition 3 Let the assumptions and the notations of Proposition 2 do apply. Then, the following statements hold true:

(i) $\Phi \circ \mathcal{A}_{u_{n}}$ is decreasing on $\left[T_{0}, n\right]$;

(ii) $\mathcal{A}_{u_{n}} \geq 0$ on $\left[T_{0}, n\right]$;

(iii) if there exists $\bar{t} \in\left[T_{0}, n\right]$ such that $\mathcal{A}_{u_{n}}(\bar{t})=0$, then

$$
\mathcal{A}_{u_{n}}(t)=0 \quad \text { for every } t \in[\bar{t}, n]
$$

(iv) $\left|\mathcal{A}_{u_{n}}\right| \leq \mathcal{N}_{L}$ on $I_{n}=[0, n]$;

(v) $\left|u_{n}^{\prime}\right| \leq \mathcal{N}_{L} / \mathbf{a}_{\star}=\gamma_{0}$ a.e. on $I_{n}$.

Proof (i) First of all, we observe that, as $\Phi \circ \mathcal{A}_{u_{n}}$ is absolutely continuous on $I_{n}$, we can prove the assertion by showing that $\left(\Phi \circ \mathcal{A}_{u_{n}}\right)^{\prime} \leq 0$ a.e. on $\left[T_{0}, n\right]$.

Now, since $u_{n}$ is a solution of the truncated problem (24) and $\alpha \leq u_{n} \leq \beta$ on $I_{n}$ (see Proposition 2-(i)), for almost every $t \in I_{n}$ we have

$$
\left(\Phi\left(\mathcal{A}_{u_{n}}(t)\right)\right)^{\prime}=\left(\Phi\left(a\left(t, u_{n}(t)\right) u_{n}^{\prime}(t)\right)\right)=f\left(t, u_{n}(t), \mathcal{D}\left(u_{n}^{\prime}\right)(t)\right) .
$$

On the other hand, since $\left|\mathcal{D}\left(u_{n}^{\prime}\right)\right| \leq \gamma_{0}=\mathcal{N}_{L} / \mathbf{a}_{\star}$ (by definition of $\mathcal{D}$ ), we are entitled to apply (15) in assumption (B4), obtaining

$$
\left(\Phi\left(\mathcal{A}_{u_{n}}(t)\right)\right)^{\prime}=f\left(t, u_{n}(t), \mathcal{D}\left(u_{n}^{\prime}\right)(t)\right) \leq 0 \text { a.e. on }\left[T_{0}, n\right] .
$$

This proves that $\Phi \circ \mathcal{A}_{u_{n}}$ is decreasing on $\left[T_{0}, n\right]$.

(ii)-(iii): With the monotonicity of $\Phi \circ \mathcal{A}_{u_{n}}$ at hand, the proof of these statements is analogous to that of [3, Proposition 3.5]; we then skip the details.

(iv) First of all, we observe that, by Proposition 2-(ii) and the explicit expression of $\mathcal{N}_{L}$ in (16), we have (remind that $K_{L} \equiv 0$ on $\left[0, T_{0}\right]$ )

$$
\left|\mathcal{A}_{u_{n}}(t)\right|<L=\mathcal{N}_{L}(t) \text { for every } t \in\left[0, T_{0}\right]
$$

moreover, by statement (ii) we know that $\mathcal{A}_{u_{n}} \geq 0$ on $\left[T_{0}, n\right]$. As a consequence, to prove the present statement we are left to demonstrate that

$$
\mathcal{A}_{u_{n}}(t) \leq \mathcal{N}_{L}(t) \text { for every } t \in\left[T_{0}, n\right]
$$


To this end, we define

$$
\widehat{t}:=\sup \left\{t \geq T_{0}: \mathcal{A}_{u_{n}}(s)<\mathcal{N}_{L}(s) \text { for all } s \in\left[T_{0}, t\right]\right\} .
$$

Since $0 \leq \mathcal{A}_{u_{n}}\left(T_{0}\right)<L=\mathcal{N}_{L}\left(T_{0}\right)$, the point $\widehat{t}$ is well defined and greater than $T_{0}$; we then argue by contradiction and we assume that $\widehat{t}<n$.

We first notice that, as a consequence of statement (iii), one has $\mathcal{A}_{u_{n}}>0$ on the whole of $\left[T_{0}, \widehat{t}\right]$ : in fact, if we have $\mathcal{A}_{u_{n}}(\bar{t})=0$ for some $\bar{t} \in\left[T_{0}, \widehat{t}\right]$, then

$(\star) \mathcal{A}_{u_{n}}<\mathcal{N}_{L}$ on $\left[T_{0}, \widehat{t}\right)$ (by definition of $\widehat{t}$ );

$(\star \star) \mathcal{A}_{u_{n}}(t)=0<\mathcal{N}_{L}$ for every $t \in[\bar{t}, n]$.

Since $\bar{t} \leq \widehat{t}$, by combining $(\star)$ with $(\star \star)$ we would obtain $\mathcal{A}_{u_{n}}<\mathcal{N}_{L}$ on $\left[T_{0}, n\right]$, which contradicts the fact that $\widehat{t}<n$. Hence,

$$
\mathcal{A}_{u_{n}}>0 \text { on the whole of }\left[T_{0}, \widehat{t}\right] \text {. }
$$

Now, since $0 \leq \mathcal{A}_{u_{n}} \leq \mathcal{N}_{L}$ on $\left[T_{0}, \widehat{t}\right]$, from (8) and Remark 3 we get

$$
0 \leq u_{n}^{\prime}(t) \leq \frac{\mathcal{N}_{L}(t)}{a\left(t, u_{n}(t)\right)} \leq \frac{\mathcal{N}_{L}}{\mathbf{a}_{\star}(t)}=\gamma_{0}(t) \quad \text { a.e. on }\left[T_{0}, \widehat{t}\right]
$$

as a consequence, bearing in mind that $u_{n}$ solves the truncated problem (24), from Proposition 2-(i) and the very definition of $\mathcal{D}$ we infer that

$$
\left(\Phi\left(\mathcal{A}_{u_{n}}(t)\right)\right)^{\prime}=\left(\Phi\left(a\left(t, u_{n}(t)\right) u_{n}^{\prime}(t)\right)\right)^{\prime}=f\left(t, u_{n}(t), u_{n}^{\prime}(t)\right) \text { a.e. on }\left[T_{0}, \widehat{t}\right] \text {. }
$$

Owing to (34) (and taking into account that $\alpha \leq u_{n} \leq \beta$ on $I_{n}$ ), we are entitled to apply estimate (13) in assumption (B4), obtaining

$$
\begin{aligned}
\left(\Phi\left(\mathcal{A}_{u_{n}}(t)\right)\right)^{\prime} & \leq-K_{L}^{\prime}(t)\left|\Phi\left(a\left(t, u_{n}(t)\right) u_{n}^{\prime}(t)\right)\right|^{\theta} \\
& \left.=-K_{L}^{\prime}(t)\left|\Phi\left(\mathcal{A}_{u_{n}}(t)\right)\right|^{\theta} \quad \text { (for a.e. } t \in\left[T_{0}, \widehat{t}\right]\right) ;
\end{aligned}
$$

in particular, since we know that $\mathcal{A}_{u_{n}}>0$ on $\left[T_{0}, \widehat{t}\right]$ (and $\Phi(0)=0$ ), we get

$$
\frac{\left(\Phi\left(\mathcal{A}_{u_{n}}(t)\right)\right)^{\prime}}{\left(\Phi\left(\mathcal{A}_{u_{n}}(t)\right)\right)^{\theta}} \leq-K_{L}^{\prime}(t) \text { for a.e. } t \in\left[T_{0}, \widehat{t}\right] .
$$

By integrating both sides of this last inequality on $\left[T_{0}, \widehat{t}\right]$, we then obtain (remind that, by assumptions, $K\left(T_{0}\right)=0$ and $\left.\theta>1\right)$

$$
\frac{1}{1-\theta}\left[\Phi\left(\mathcal{A}_{u_{n}}(\widehat{t})\right)^{1-\theta}-\Phi\left(\mathcal{A}_{u_{n}}\left(T_{0}\right)\right)^{1-\theta}\right] \leq-K_{L}(\widehat{t}) .
$$

As a consequence, since $\mathcal{A}_{u_{n}}\left(T_{0}\right)<L$ and $a$ is nonnegative, we conclude that

$$
\Phi\left(\mathcal{A}_{u_{n}}(\widehat{t})\right)<\Phi^{-1}\left\{\left(\Phi(L)^{1-\theta}+(\theta-1) K_{L}(\widehat{t})\right)^{1 /(1-\theta)}\right\}=\mathcal{N}_{L}(\widehat{t})
$$

This is in contradiction with the definition of $\widehat{t}$, and thus $\widehat{t}=n$.

(iv) It is a direct consequence of statement (iv) and Proposition 2-(i), taking also into account Remark 3. This ends the proof.

Gathering together Propositions 2 and 3, we obtain the following result. 
Theorem 3 In the above assumptions and notations, let $u_{n} \in W^{1, p}\left(I_{n}\right)$ be any solution of the truncated problem (24). Then $u_{n}$ is a solution of $\left(P_{n}\right)$.

Proof Since $u_{n}$ solves (on $I_{n}$ ) the BVP (24), we clearly have

$$
u_{n}(0)=\alpha(0) \text { and } u_{n}(n)=\beta(n)
$$

furthermore, taking into account the very definitions of $\mathcal{T}, \mathcal{D}$ and $f^{*}$ and combining Proposition 2-(i) with Proposition 3-(v) we obtain

$$
\begin{aligned}
\left(\Phi\left(a\left(t, u_{n}(t)\right) u_{n}^{\prime}(t)\right)\right)^{\prime} & =\left(\Phi\left(a\left(t, \mathcal{T}\left(u_{n}\right)(t)\right) u_{n}^{\prime}(t)\right)\right)^{\prime} \\
& =f^{*}\left(t, u_{n}(t), \mathcal{D}\left(\mathcal{T}\left(u_{n}\right)^{\prime}(t)\right)\right)=f\left(t, u_{n}(t), u_{n}^{\prime}(t)\right) .
\end{aligned}
$$

Hence, $u_{n}$ solves $\left(P_{n}\right)$, as claimed. This ends the proof.

\section{From $[0, n]$ to $[0, \infty)$ : the proof of Theorem 1}

Throughout the sequel, we take for fixed all the assumptions and the notation introduced in Sects. 2 and 3.

As anticipated at the end of Sect. 2, the aim of the present section is to prove the existence result in Theorem 1; to this end, we shall use a suitable limit argument involving the solutions of the (family of) ODEs (with $n>T_{0}$ )

$$
\left(\Phi\left(a(t, x(t)) x^{\prime}(t)\right)\right)^{\prime}=f\left(t, x(t), x^{\prime}(t)\right) \text { for a.e. } t \in[0, n] .
$$

Since this argument is very similar to that already exploited in the demonstration of [3, Theorem 2.4], we limit ourselves to sketch the key steps toward the proof of our Theorem 1 and we directly refer to [3] for all the details.

Proof of Theorem 1 Let $T_{0}>0$ be as in assumptions (B1)-(B4); for every $n>T_{0}$, we let $I_{n}:=[0, n]$ and we choose a solution $u_{n} \in W^{1, p}\left(I_{n}\right)$ of (24). By Theorem $3, u_{n}$ solves $\left(P_{n}\right)$ on $I_{n}$; moreover, by Proposition 3 we get

$$
\begin{aligned}
& (\star)\left|\mathcal{A}_{u_{n}}(t)\right| \leq \mathcal{N}_{L}(t) \text { for every } t \in I_{n} \text { (here, } L \text { is as in (22)); } \\
& (\star)\left|u_{n}^{\prime}(t)\right| \leq \mathcal{N}_{L}(t) / \mathbf{a}_{\star}(t) \text { for almost every } t \in I_{n} \text {. }
\end{aligned}
$$

We then consider the sequence $\left\{x_{n}\right\}_{n} \subseteq W^{1, p}(\Lambda)$ defined as follows:

$$
x_{n}: \Lambda \rightarrow \mathbb{R}, \quad x_{n}(t)= \begin{cases}u_{n}(t), & \text { if } t \in I_{n}=[0, n], \\ \beta(n), & \text { if } t>n .\end{cases}
$$

We claim that $\left\{x_{n}\right\}_{n}$ uniformly converges (up to a subsequence) to a function $x_{0} \in W_{\text {loc }}^{1, p}(\Lambda)$, which turns out to be a solution of (17). The proof of this claim relies on some ideas already used in [3] and it articulates in the following steps.

STEP I: For every $n>T_{0}$, we consider set 


$$
\begin{aligned}
& y_{n}(t):=\left\{\begin{array}{ll}
u_{n}^{\prime}(t), & \text { if } t \in I_{n} \text { and } \exists u_{n}^{\prime}(t), \\
0, & \text { otherwise; }
\end{array}\right. \text { and } \\
& z_{n}(t):= \begin{cases}\left(\Phi\left(\mathcal{A}_{u_{n}}(t)\right)\right)^{\prime}, & \text { if } t \in I_{n} \text { and } \exists\left(\Phi \circ \mathcal{A}_{u_{n}}\right)^{\prime}(t), \\
0, & \text { otherwise. }\end{cases}
\end{aligned}
$$

Since $u_{n}$ is a solution of $\left(P_{n}\right)$, it can be easily deduced from assumption (B4), (36) and Remark 3 that both the sequences $\left\{y_{n}\right\}_{n}$ and $\left\{z_{n}\right\}_{n}$ are uniformly integrable in $L^{1}(\Lambda)$; as a consequence, the Dunford-Pettis theorem (see, e.g., [5]) provides us with two functions $g, h \in L^{1}(\Lambda)$ such that (up to a subsequence)

$$
y_{n} \rightarrow g \text { and } z_{n} \rightarrow h \text { in } L^{1}(\Lambda) \text { as } n \rightarrow \infty .
$$

Now, since $x_{n}(0)=\alpha(0)$ for every $n>T_{0}$, from (37) we get

$$
x_{n}(t)=x_{n}(0)+\int_{0}^{t} x_{n}^{\prime}(s) \mathrm{d} s \underset{n \rightarrow \infty}{\longrightarrow} \alpha(0)+\int_{0}^{t} g(s) \mathrm{d} s=: x_{0}(t) \quad \forall t \in \Lambda .
$$

Furthermore, since $\left\{\mathcal{A}_{u_{n}}(0)\right\}_{n}$ is bounded (by Proposition 2-(ii)) and since there exists a null set $E \subseteq \Lambda$ such that, for $n>T_{0}$ and $t \in I_{n} \backslash E$,

$$
\Phi\left(a\left(t, x_{n}(t)\right) x_{n}^{\prime}(t)\right)=\Phi\left(\mathcal{A}_{u_{n}}(t)\right)=\Phi\left(\mathcal{A}_{u_{n}}(0)\right)+\int_{0}^{t} z_{n}^{\prime}(s) \mathrm{d} s,
$$

again from (37) we infer that, for every $t \in \Lambda \backslash E$, one has

$$
a\left(t, x_{n}(t)\right) x_{n}^{\prime}(t) \underset{n \rightarrow \infty}{\longrightarrow} \Phi^{-1}\left(\Phi(v)+\int_{0}^{t} h(s) \mathrm{d} s\right)=: \mathcal{U}(t),
$$

where $v$ is a suitable real number such that $\mathcal{A}_{u_{n}}(0) \rightarrow v$ as $\left.n \rightarrow \infty\right)$. Gathering together (38), (39) and the continuity of $a$, we obtain (see also Remark 3-(i))

$$
y_{n}(t)=x_{n}^{\prime}(t) \underset{n \rightarrow \infty}{\longrightarrow} \frac{1}{a\left(t, x_{0}(t)\right)} \mathcal{U}(t) \text { for a.e. } t \in \Lambda .
$$

On the other hand, since the limit in (40) also holds in $L^{1}(\Lambda)$ (as a simple dominated convergence argument shows), from (37) we infer that

$$
x_{0}^{\prime}(t) \stackrel{(38)}{=} g(t)=\frac{1}{a\left(t, x_{0}(t)\right)} \mathcal{U}(t) \quad \text { for a.e. } t \in \Lambda ;
$$

taking into account the very definition of $\mathcal{U}$, by (41) we then conclude that

(a) $x_{0}^{\prime} \in L_{\text {loc }}^{p}(\Lambda)$, whence $x_{0} \in W_{\text {loc }}^{1, p}(\Lambda)$ (see Remark 3);

(b) $a\left(t, x_{0}\right) x_{0}^{\prime}=\mathcal{U}$ a.e. on $\Lambda$;

(c) $\Phi \circ\left(a\left(t, x_{0}\right) x_{0}^{\prime}\right)=\Phi \circ \mathcal{U} \in W_{\mathrm{loc}}^{1,1}(\Lambda)$ and $\left(\Phi\left(a\left(t, x_{0}\right) x_{0}^{\prime}\right)\right)^{\prime}=h$.

We also notice that, as a by-product of (40) and (41), it holds that

$$
\lim _{n \rightarrow \infty} x_{n}^{\prime}=x_{0} \quad \text { both in } L^{1}(\Lambda) \text { and a.e. on } \Lambda \text {. }
$$

STEP II: In this step, we prove that $x_{0}$ solves the ODE in (17). To this end, we first observe that, since $u_{n}$ solves $\left(P_{n}\right)$ on $I_{n}$, there exists another null set $F \subseteq \Lambda$ such that, for $n>T_{0}$ and $t \in I_{n} \backslash F$,

$$
z_{n}(t)=\left(\Phi\left(a\left(t, u_{n}(t)\right) u_{n}^{\prime}(t)\right)\right)^{\prime}=f\left(t, u_{n}(t), u_{n}^{\prime}(t)\right)=f\left(t, x_{n}(t), x_{n}^{\prime}(t)\right) ;
$$


from this, by using (38) and (42) we get

$$
\lim _{n \rightarrow \infty} z_{n}(t)=f\left(t, x_{0}(t), x_{0}^{\prime}(t)\right) \text { for a.e. } t \in \Lambda .
$$

On the other hand, since the above limit also holds in $L^{1}(\Lambda)$ (as another dominated convergence argument shows, see (14)), from (37) we obtain

$$
\left(\Phi\left(a\left(t, x_{0}(t)\right) x_{0}^{\prime}(t)\right)\right)^{\prime} \stackrel{(\mathrm{c})}{=} h(t)=f\left(t, x_{0}(t), x_{0}^{\prime}(t)\right) \text { for a.e. } t \in \Lambda
$$

which proves that $x_{0}$ is a solution of the ODE in (17).

STEP III: Finally, by (42) and the fact that $x_{n}(0)=\alpha(0)$ we have

$$
\sup _{\Lambda}\left\|x_{n}-x_{0}\right\| \leq\left\|x_{n}^{\prime}-x_{0}^{\prime}\right\|_{L^{1}(\Lambda)} ;
$$

as a consequence, we derive that $x_{n} \rightarrow x_{0}$ uniformly on $\Lambda$, whence

$$
\lim _{t \rightarrow \infty} x_{0}(t)=\lim _{n \rightarrow \infty}\left(\lim _{t \rightarrow \infty} x_{n}(t)\right)=\lim _{n \rightarrow \infty} \beta(n)=\lim _{t \rightarrow \infty} \beta(t)=\beta_{0} .
$$

This proves that $x_{0}$ is a solution of (5).

\section{A class of problems}

The aim of this last section is to present a wide class of boundary value problems to which our existence result in Theorem 1 applies; we also provide some concrete examples of problems falling in this class.

Let $v_{1}, v_{2} \in \mathbb{R}$ be arbitrary real numbers such that $v_{1}<v_{2}$. Throughout the sequel we consider boundary value problems of the following form

$$
\left\{\begin{array}{l}
\left(\Phi\left(a(t, x(t)) x^{\prime}(t)\right)\right)^{\prime}=f_{1}(t, x(t)) f_{2}\left(x^{\prime}(t)\right) \text { a.e. on } \Lambda \\
x(0)=v_{1}, \quad x(\infty)=v_{2},
\end{array}\right.
$$

where the functions $a, \Phi, f_{1}$ and $f_{2}$ satisfy the assumptions listed as follows:

(I) $a: \Lambda \times \mathbb{R} \rightarrow \mathbb{R}$ is a nonnegative continuous function on $\Lambda \times \mathbb{R}$ which is also bounded on $\Lambda \times\left[v_{1}, v_{2}\right]$. Furthermore, we suppose that it is possible to find a real $p>1$ and a real $\sigma>1$ such that, setting

$$
h(t):=\min _{x \in\left[v_{1}, v_{2}\right]} a(t, x) \geq 0,
$$

we have $1 / h \in L_{\text {loc }}^{p}(\Lambda)$ and

$$
\int_{1}^{\infty} \frac{1}{t^{\sigma} h(t)^{p}} \mathrm{~d} t<\infty
$$

(II) $\Phi: \mathbb{R} \rightarrow \mathbb{R}$ is an odd function fulfilling assumption (A1) in Sect. 2 (with a suitable $\rho>0$ ); hence, in particular, we have

$$
\liminf _{z \rightarrow 0^{+}} \frac{\Phi(z)}{z^{\rho}}>0 .
$$

(III) $f_{1}: \Lambda \times \mathbb{R} \rightarrow \mathbb{R}$ is a Carathéodory function enjoying the following properties: 
(III) $)_{1}$ there exists a function $\lambda \in L_{\mathrm{loc}}^{\infty}(\Lambda)$ such that

$$
\left|f_{1}(t, x)\right| \leq \lambda(t) \quad \text { for a.e. } t \in \mathbb{R} \text { and every } x \in\left[v_{1}, v_{2}\right] ;
$$

(III) $)_{2}$ it is possible to find a real number $T_{0}>0$, two real constants $c_{1}, c_{2}>0$ and two numbers $\delta_{1}, \delta_{2} \in \mathbb{R}$, with $-1<\delta_{1} \leq \delta_{2}$, such that

$$
c_{1} t^{\delta_{1}} \leq\left|f_{1}(t, x)\right| \leq c_{2} t^{\delta_{2}},
$$

for a.e. $t \geq T_{0}$ and every $x \in\left[v_{1}, v_{2}\right]$;

(III) $3 f_{1}(t, x) \leq 0$ for every $t \geq T_{0}$ and every $x \in\left[v_{1}, v_{2}\right]$;

(IV) $f_{2} \in C(\mathbb{R}, \mathbb{R})$ and it enjoys the following properties:

(IV) $1 f_{2}>0$ on $\mathbb{R} \backslash\{0\}$ and $f_{2}(0)=0$;

$(\mathrm{IV})_{2}$ there exist a real number $y^{\star}>0$, two real constants $c_{1}^{\prime}, c_{2}^{\prime}>0$ and two numbers $\gamma_{1}, \gamma_{2} \in \mathbb{R}$, with $\gamma_{2} \leq \gamma_{1}$ and $\gamma_{1}>1$, such that

$$
c_{1}^{\prime}|\Phi(y)|^{\gamma_{1}} \leq f_{2}(y) \leq c_{2}^{\prime}|\Phi(y)|^{\gamma_{2}},
$$

for every $y \in \mathbb{R}$ with $|y|<y^{\star}$;

$(\mathrm{IV})_{3}$ there exists a strictly increasing function $\omega:(0, \infty) \rightarrow(0, \infty)$ satisfying the following properties (here $p>1$ is as in assumption (I)):

(i) $\liminf _{s \rightarrow 0^{+}}\left(s^{-p} \omega(s)\right)>0$;

(ii) $f_{2}(s y)=\omega(s) f_{2}(y)$ for every $s>0$ and every $y \in \mathbb{R}$.

$(\mathrm{IV})_{4}$ there exist a real $H>0$ and a real constant $c_{3}^{\prime}>0$ such that, if $y \in \mathbb{R}$ and $|y| \geq H$, the following estimate holds true:

$$
f_{2}(y) \leq c_{3}^{\prime}|\Phi(y)|^{\alpha} \quad \text { for some } \alpha \in(0,1] .
$$

Furthermore, we suppose that the following relations hold among all the constants appearing in assumptions (I)-(IV):

$$
\frac{\delta_{1}+1}{\gamma_{1}-1} \geq \sigma \rho \quad \text { and } \quad \frac{\gamma_{2}\left(\delta_{1}+1\right)}{\gamma_{1}-1} \geq \sigma+\delta_{2} .
$$

Our aim is to prove that, in the present setting, all the hypotheses of Theorem 1 are satisfied; as a consequence, there exists a solution $x \in W_{\text {loc }}^{1, p}(\Lambda)$ of (43).

Remark 7 Before proceeding we highlight, for a future reference, a few consequences of assumptions (I)-(IV) we shall use in the sequel.

1. If $h$ and $\omega$ are, respectively, as in assumptions (I) and (IV) $)_{3}$, we have

$$
t \mapsto 1 / \omega(h(t)) \in L_{\mathrm{loc}}^{1}(\Lambda) .
$$

In fact, on account of (49), it is possible to find a constant $\mathbf{c}>0$ such that

$$
\omega(s) \geq \mathbf{c} s^{p}, \quad \text { for every } 0 \leq s \leq 1 ;
$$

as a consequence, since $1 / h \in L_{\mathrm{loc}}^{p}(\Lambda)$, for every $T>0$ we obtain

$$
\begin{aligned}
\int_{0}^{T} \frac{1}{\omega(h(t))}= & \left(\int_{\{h>1\}}+\int_{\{h \leq 1\}}\right) \frac{1}{\omega(h(t))} \mathrm{d} t \\
& (\text { since } \omega \text { is strictly increasing) }
\end{aligned}
$$




$$
\leq \frac{T}{\omega(1)}+\frac{1}{\mathbf{c}} \int_{0}^{T} \frac{1}{h(t)^{p}} \mathrm{~d} t<\infty .
$$

2. For every $v \geq \sigma$, one has

$$
\int_{1}^{\infty} \frac{1}{t^{v} \omega(h(t))} \mathrm{d} t<\infty .
$$

This assertion can be demonstrated by arguing exactly as above, taking into account (45) and the fact that, by assumption, $\sigma>1$.

3. For every $v \leq p$, one has

$$
\int_{1}^{\infty} \frac{1}{t^{\sigma} h(t)^{v}} \mathrm{~d} t<\infty
$$

In fact, since $a(t, x) \geq h(t)$ for any $(t, x) \in \Lambda \times\left[v_{1}, v_{2}\right]$ and $a$ is bounded on the same set, we have that $h$ is bounded on $\Lambda$; as a consequence, since the map $t \mapsto t^{-\sigma} / h^{p}$ is integrable on $(1, \infty)$, for every $v \in(-\infty, p]$ we have

$$
\int_{1}^{\infty} \frac{1}{t^{\sigma} h(t)^{v}} \mathrm{~d} t \leq\left(\sup _{\Lambda} h\right)^{p-v} \cdot \int_{1}^{\infty} \frac{1}{t^{\sigma} h(t)^{p}} \mathrm{~d} t<\infty .
$$

4. For every $\zeta>0$, one has

$$
\max _{|y| \leq \zeta}|\Phi(y)|=\Phi(\zeta)
$$

In fact, if $y \in \mathbb{R}$ is such that $|y| \leq \zeta$, from the fact that $\Phi$ is an odd increasing function on $\mathbb{R}$ (see assumption (II)) we infer that

$$
-\Phi(\zeta)=\Phi(-\zeta) \leq \Phi(y) \leq \Phi(\zeta), \text { whence }|\Phi(y)| \leq \Phi(\zeta)
$$

We now prove that all the hypotheses of Theorem 1 are satisfied.

Hypotheses (A1)-(A3). We begin by showing that, setting

$$
f: \Lambda \times \mathbb{R}^{2} \rightarrow \mathbb{R}, \quad f(t, x, y):=f_{1}(t, x) f_{2}(y),
$$

then the functions $\Phi, a$ and $f$ satisfy hypotheses (A1)-(A3) in Sect. 2.

In fact, on account of assumptions (I) and (II), we have that $\Phi$ satisfies (A1) (with a suitable $\rho>0$ ), whereas $a$ satisfies (A2); furthermore, since $f_{1}$ is a Carathéodory function on $\Lambda \times \mathbb{R}$ and $f_{2}$ is continuous on $\mathbb{R}$ (as it follows from assumptions (III) and (IV)), we conclude that $f$ satisfies (A3).

Hypothesis (B1). We now prove that hypothesis (B1) in the statement of Theorem 1 is satisfied. To this end, we observe that, since $f_{2}(0)=0$ (as it follows from assumption (IV) 1 ), the (constant) functions

$$
\alpha(t)=v_{1} \text { and } \beta(t)=v_{2} \quad(t \in \Lambda)
$$

are, respectively, a lower solution and an upper solution of the ODE

$$
\left(\Phi\left(a(t, x(t)) x^{\prime}(t)\right)\right)^{\prime}=f_{1}(t, x(t)) f_{2}\left(x^{\prime}(t)\right)=f\left(t, x(t), x^{\prime}(t)\right),
$$

according to Definition (see also Remark 2-(2)). Moreover, we clearly have

$-\alpha \leq \beta$ on $\Lambda$ (since $\left.v_{1}<v_{2}\right)$;

$-\beta$ is increasing on $\left[T_{0}, \infty\right)$ (where $T_{0}>0$ is as in assumption (III));

$-\beta \rightarrow v_{2}=\beta_{0} \in \mathbb{R}$ as $t \rightarrow \infty$. 
Hypothesis (B2). In this paragraph, we prove that $a$ satisfies assumption (B2). In fact, since the (constant) functions $\alpha(t) \equiv v_{1}$ and $\beta(t) \equiv v_{2}$ are, respectively, a lower solution and an upper solution of the ODE (58), we have (see (9))

$$
\mathbf{a}_{\star}(t)=\min _{x \in[\alpha(t), \beta(t)]} a(t, x)=h(t) \text { for every } t \in \Lambda .
$$

As a consequence, from assumption (I) we infer that $1 / \mathbf{a}_{\star}=1 / h \in L_{\text {loc }}^{p}(\Lambda)$ (for a suitable $p>1$ ), whence hypothesis (B2) is fulfilled.

Hypothesis (B3). In this paragraph, we prove that also hypothesis (B3) is satisfied. In fact, if $H>0$ is as in assumption (IV) $)_{4}$, by gathering together (51), (46) and (50) we obtain the following estimate

$$
\begin{aligned}
|f(t, x, y)|= & \left|f_{1}(t, x)\right| \cdot\left|f_{2}(y)\right|=\frac{\left|f_{1}(t, x)\right|}{\omega(a(t, x))}\left|f_{2}(a(t, x) y)\right| \\
\leq & c_{3}^{\prime} \frac{\lambda(t)}{\omega(a(t, x))}|\Phi(a(t, x) y)|^{\alpha} \\
& (\text { by }(44) \text { and the monotonicity of } \omega) \\
\leq & c_{3}^{\prime} \frac{\lambda(t)}{\omega(h(t))}|\Phi(a(t, x) y)|^{\alpha},
\end{aligned}
$$

holding true for a.e. $t \in \mathbb{R}$, any $x \in\left[v_{1}, v_{2}\right]$ and any $y \in \mathbb{R}$ with $|a(t, x) y| \geq H$. As a consequence, hypothesis (B3) is fulfilled with the choice

$$
\psi(s)=s^{\alpha}, \quad l(t)=c_{3}^{\prime} \frac{\lambda(t)}{\omega(h(t))}, \quad \mu(t) \equiv 0,
$$

and $T_{0}>0$ as in assumption (III). We explicitly point out that, since $\alpha \leq 1$, the function $\psi$ satisfies (10); furthermore, since $\lambda$ belongs to $L_{\text {loc }}^{\infty}(\Lambda)$, it readily follows from (53) in Remark 3 that $l \in L^{1}\left(\left[0, T_{0}\right]\right)$.

Hypothesis (B4). In this last paragraph of the section, we prove that, in our setting, hypothesis (B4) in the statement of Theorem 1 is satisfied.

To begin with, if $T_{0}>0$ is as in assumption (III), we consider the function

$$
K_{0}: \Lambda \longrightarrow \mathbb{R}, \quad K_{0}(t):= \begin{cases}0, & \text { if } 0 \leq t \leq T_{0} \\ \int_{T_{0}\left[\nu_{1}, \nu_{2}\right]}^{t} \min _{1}\left|f_{1}(s, \cdot)\right| \mathrm{d} s, & \text { if } t>T_{0} .\end{cases}
$$

Moreover, given any $L>0$, we set

- $M_{a}:=\sup _{\Lambda \times\left[\nu_{1}, v_{2}\right]} a<\infty \quad$ (see assumption (I));

- $m_{f_{2}}(L):=\min _{y^{\star} \leq|y| \leq L} f_{2}(y) \quad\left(\right.$ where $y^{\star}$ is as in assumption $\left.(\mathrm{IV})_{2}\right)$;

- $M_{\Phi}(L):=\max _{y^{\star} \leq|z| \leq L}|\Phi(z)|^{\gamma_{1}} \quad\left(\text { where } \gamma_{1} \text { is as in assumption (IV) }\right)_{2}$ ).

Finally, if $c_{1}^{\prime}>0$ is as in (48) and if $\omega$ is the function appearing in assumption (IV) $)_{3}$, we consider the function $K_{L}$ defined on $\Lambda$ by

$$
K_{L}(t):=\mathbf{c}(L) \cdot K_{0}(t), \quad \text { where } \mathbf{c}(L):=\min \left\{\frac{c_{1}^{\prime}}{\omega\left(M_{a}\right)}, \frac{m_{f_{2}}(L)}{M_{\Phi}(L) \omega\left(M_{a}\right)}\right\} .
$$

Now, by arguing exactly as in [3, Section 5] (where the same function $K_{0}$ is considered, but multiplied times a different $\mathbf{c}(L))$, one can recognize that 
(i) $K_{L} \in W_{\mathrm{loc}}^{1,1}(\Lambda)$ and $K_{L} \equiv 0$ on $\left[0, T_{0}\right]$;

(ii) $K_{L}$ is strictly increasing on $\left[T_{0}, \infty\right)$ and $K_{L} \rightarrow \infty$ as $t \rightarrow \infty$;

(iii) $K_{L}$ satisfies the integrability property (12) with $\theta=\gamma_{1}>1$, that is,

$$
\int_{T_{0}+1}^{\infty} \frac{1}{\mathbf{a}_{\star}(t)} K_{0}(t)^{-\frac{1}{\rho\left(\gamma_{1}-1\right)}} \mathrm{d} t=\int_{T_{0}+1}^{\infty} \frac{1}{h(t)} K_{0}(t)^{-\frac{1}{\rho\left(\gamma_{1}-1\right)}} \mathrm{d} t<\infty .
$$

(It should be noticed that, in proving (i)-(iii), assumptions (I)-(III) and the first condition in (52) play a crucial rôle.) Furthermore, by taking into account that (see (16) with $\theta=\gamma_{1}$ )

$$
0<\mathcal{N}_{L}=\Phi^{-1}\left\{\left(\Phi(L)^{1-\gamma_{1}}+\left(\gamma_{1}-1\right) K_{L}\right)^{1 /\left(1-\gamma_{1}\right)}\right\} \leq L \text { on } \Lambda,
$$

we also have the following estimate, holding true for almost every $t \geq T_{0}$, every $x \in\left[v_{1}, v_{2}\right]$ and every $y \in \mathbb{R}$ with $|y| \leq \mathcal{N}_{L}(t) / a(t, x)$ :

$$
\begin{aligned}
& f_{1}(t, x) f_{2}(y) \\
&=\frac{f_{1}(t, x)}{\omega(a(t, x))} \omega(a(t, x)) f_{2}(y) \stackrel{(50)}{=} \frac{f_{1}(t, x)}{\omega(a(t, x))} f_{2}(a(t, x) y) \\
&\text { (by (I), (III) } \left.)_{3} \text { and the monotonicity of } \omega\right) \\
& \leq \frac{1}{\omega\left(M_{a}\right)} f_{1}(t, x) f_{2}(a(t, x) y) \\
& \leq\left\{\begin{array}{l}
\frac{c_{1}^{\prime}}{\omega\left(M_{a}\right)} f_{1}(t, x)|\Phi(a(t, x) y)|^{\gamma_{1}}, \quad \text { if }|a(t, x) y| \leq y^{\star} \quad(\text { by }(48)), \\
\left(\frac{m_{f_{2}}(L)}{M_{\Phi}(L) \omega\left(M_{a}\right)}\right) f_{1}(t, x)|\Phi(a(t, x) y)|^{\gamma_{1}}, \quad \text { if } y^{\star} \leq|a(t, x) y| \leq \mathcal{N}_{L}(t) .
\end{array}\right.
\end{aligned}
$$

As a consequence, by the very definition of $\mathbf{c}(L)$ and $K_{L}$ we get

$$
\begin{aligned}
f(t, x, y) & =f_{1}(t, x) f_{2}(y) \leq \mathbf{c}(L) f_{1}(t, x)|\Phi(a(t, x) y)|^{\gamma_{1}} \\
& \leq\left(-\mathbf{c}(L) \min _{\left[v_{1}, v_{2}\right]}\left|f_{1}(t, \cdot)\right|\right)|\Phi(a(t, x) y)|^{\gamma_{1}} \\
& =-K_{L}^{\prime}(t)|\Phi(a(t, x) y)|^{\gamma_{1}}
\end{aligned}
$$

and this proves that also property (13) is fulfilled (again with $\theta=\gamma_{1}$ ).

To conclude the demonstration of the validity of hypothesis (B4), we are left to show that conditions (14) and (15) hold (with a suitable $\eta_{L} \in L^{1}(\Lambda)$ ).

First of all, since $f_{1}<0$ a.e. on $\left[T_{0}, \infty\right) \times \Lambda$ and $f_{2} \geq 0$ on the whole of $\mathbb{R}$ (see, respectively, assumptions (III) $)_{3}$ and (IV) 1 ), we obviously have

$$
f(t, x, y)=f_{1}(t, x) f_{2}(y) \leq 0 \text { for } t \geq T_{0} \text {, every } x \in\left[v_{1}, v_{2}\right] \text { and every } y \in \mathbb{R} ;
$$

we then turn to prove the existence of a nonnegative function $\eta_{L} \in L^{1}(\Lambda)$ such that (see (59) and take into account Remark 2-(3))

$$
|f(t, x, y)| \leq \eta_{L}(t) \text { for a.e. } t \geq T_{0} \text {, every } x \in\left[v_{1}, v_{2}\right] \text { and }|y| \leq \mathcal{N}_{L}(t) / h(t) .
$$

To begin with, we observe that, since $K_{L} \rightarrow \infty$ as $t \rightarrow \infty$ (see (b) above), by the very definition of $\mathcal{N}_{L}$ and the fact that $\Phi(0)=0$ we get

$$
\lim _{t \rightarrow \infty} \mathcal{N}_{L}(t)=0 .
$$


In particular, it is possible to find a real $\bar{t}_{L}>T_{0}$ such that

$$
0<\mathcal{N}_{L}(t)<y^{\star} \text { for every } t \geq \bar{t}_{L} .
$$

As a consequence, for almost every $t \in \Lambda$, every $x \in\left[v_{1}, v_{2}\right]$ and every $y \in \mathbb{R}$ such that $|h(t) y| \leq \mathcal{N}_{L}(t)$ we have the following estimate:

$$
\begin{aligned}
|f(t, x, y)| & =\left|f_{1}(t, x)\right| f_{2}(y)=\frac{\left|f_{1}(t, x)\right|}{\omega(h(t))} f_{2}(h(t) y) \quad(\text { by }(50)) \\
& \leq\left\{\begin{array}{lll}
c_{2} c_{2}^{\prime} \frac{t^{\delta_{2}}}{\omega(h(t))} \Phi\left(\mathcal{N}_{L}(t)\right)^{\gamma_{2}}, & \text { if } t>\bar{t}_{L} & \text { (by (47), (48) and Rem. 7-(b)), } \\
\left(\max _{[0, L]} f_{2}\right) \cdot \frac{\lambda(t)}{\omega(h(t))}, & \text { if } t \leq \bar{t}_{L} & \text { (by (46)), } \\
& =: \eta_{L}(t) .
\end{array}\right.
\end{aligned}
$$

To complete the demonstration of the validity of assumption (B4), we are left to prove that $\eta_{L} \in L^{1}(\Lambda)$. To this end, we first observe that, since $\lambda \in L_{\mathrm{loc}}^{\infty}(\Lambda)$ (see assumption (III)), it readily follows from (53) that

$$
\int_{0}^{\bar{t}_{L}} \eta_{L}(t) \mathrm{d} t<\infty
$$

on the other hand, by proceeding as in [3, Section 5] (where the same $\mathcal{N}_{L}$ is considered, apart from a different constant in the definition of $K_{L}$ ) one gets

$$
\begin{aligned}
\Phi\left(\mathcal{N}_{L}(t)\right)^{\gamma_{2}} & \stackrel{(16)}{=}\left(\Phi(L)^{1-\gamma_{1}}+\left(\gamma_{1}-1\right) \mathbf{c}(L) \cdot K_{0}\right)^{\gamma_{2} /\left(1-\gamma_{1}\right)} \\
& \leq \mathbf{c}\left(t^{\delta_{1}+1}-T_{0}^{\delta_{1}+1}\right)^{-\gamma_{2} /\left(\gamma_{1}-1\right)} \\
& \leq \mathbf{c}^{\prime} t^{-\frac{\gamma_{2}\left(\delta_{1}+1\right)}{\gamma_{1}-1}}, \quad \text { for every } t>\bar{t}_{L}>T_{0},
\end{aligned}
$$

for suitable constants $\mathbf{c}, \mathbf{c}^{\prime}>0$. From this, since (52) obviously implies that

$$
\frac{\gamma_{2}\left(\delta_{1}+1\right)}{\gamma_{1}-1}-\delta_{2} \geq \sigma
$$

we finally obtain (see also (54) in Remark 7-(a))

$$
\int_{\bar{t}_{L}}^{\infty} \eta_{L}(t) \mathrm{d} t \leq\left(c_{2} c_{2}^{\prime} \mathbf{c}^{\prime}\right) \int_{\bar{t}_{L}}^{\infty} \frac{1}{\omega(h(t))} t^{\delta_{2}-\frac{\gamma_{2}\left(\delta_{1}+1\right)}{\gamma_{1}-1}} \mathrm{~d} t<\infty .
$$

This completes the proof of the validity of assumption (B4).

Remark 8 It is worth observing that, if condition (49) in assumption (IV) $)_{3}$ holds true in the following stronger form (remind that $p>1$ )

$$
\liminf _{s \rightarrow 0^{+}} \frac{\omega(s)}{s^{\tau}}>0 \text { for some } \tau \in(0,1],
$$

then estimate (51) in assumption (IV) 4 can be replaced by the one as follows:

(IV) ${ }_{4}^{\prime}$ there exist a real $H>0$ and a real constant $c_{3}^{\prime}>0$ such that, if $y \in \mathbb{R}$ and $|y| \geq H$, the following estimate holds:

$$
f_{2}(y) \leq c_{3}^{\prime}|y|^{1-1 / q} \quad \text { for some } q \geq \frac{1}{1-\tau} .
$$


As usual, if (60) holds with $\tau=1$, we require (61) to hold with $q=\infty$, with the convention that $1 / q=0$.

In fact, let us assume that both (60) and (61) are satisfied; we then have

$$
|f(t, x, y)|=\left|f_{1}(t, x)\right| \cdot\left|f_{2}(y)\right| \stackrel{(50)}{=} \frac{\left|f_{1}(t, x)\right|}{\omega(a(t, x))}\left|f_{2}(a(t, x) y)\right|
$$

(by assumption (III) $\left.)_{1}\right)$

$$
\begin{aligned}
& \leq c_{3}^{\prime} \frac{\lambda(t)}{\omega(a(t, x))}|a(t, x) y|^{1-1 / q} \\
& =c_{3}^{\prime} \lambda(t)\left[\frac{a(t, x)^{1-1 / q}}{\omega(a(t, x))}\right]|y|^{1-1 / q},
\end{aligned}
$$

for a.e. $t \in \mathbb{R}$, every $x \in\left[v_{1}, v_{2}\right]$ and every $y \in \mathbb{R}$ with $|a(t, x) y| \geq H$. As a consequence, if we are able to demonstrate the existence of a suitable $\Omega>0$

$$
0 \leq \frac{a(t, x)^{1-1 / q}}{\omega(a(t, x))} \leq \Omega, \text { for a.e. }(t, x) \in \Lambda \times\left[v_{1}, v_{2}\right],
$$

we can conclude that hypothesis (B3) in Theorem 1 is again fulfilled with the choice (remember that, by assumption, $\lambda \in L_{\mathrm{loc}}^{\infty}(\Lambda)$ )

$$
\psi(s) \equiv 1, \quad l(t) \equiv 0, \quad \mu(t)=\Omega c_{3}^{\prime} \lambda(t),
$$

and $T_{0}>0$ as in assumption (III). To prove (62), we argue as follows: On the one hand, since $\omega$ is strictly increasing and the function $a$ is bounded on $\Lambda \times\left[v_{1}, v_{2}\right]$, for every $(t, x) \in$ $\Lambda \times\left[v_{1}, v_{2}\right]$ such that $a(t, x) \geq 1$ we have

$$
0 \leq \frac{a(t, x)^{1-1 / q}}{\omega(a(t, x))} \leq \frac{\left(M_{a}\right)^{1-1 / q}}{\omega(1)}
$$

on the other hand, since (60) implies the existence of a constant $\mathbf{c}>0$ such that

$$
\omega(s) \geq \mathbf{c} s^{\tau} \text { for every } 0<s \leq 1,
$$

for every $(t, x) \in \Lambda \times\left[v_{1}, v_{2}\right]$ with $0<a(t, x)<1$ we obtain

$$
\begin{aligned}
0 & \leq \frac{a(t, x)^{1-1 / q}}{\omega(a(t, x))} \leq \frac{1}{\mathbf{c}} a(t, x)^{1-1 / q-\tau} \\
& (\text { since, by the choice of } q, \text { we have } 1-1 / q-\tau \geq 0) \\
& \leq \frac{1}{\mathbf{c}}\left(M_{a}\right)^{1-1 / q-\tau} .
\end{aligned}
$$

Taking into account that $a>0$ almost everywhere on $\Lambda \times \mathbb{R}$ (see Remark 3), we conclude that the claimed (62) holds with the choice

$$
\Omega:=\max \left\{\frac{\left(M_{a}\right)^{1-1 / q}}{\omega(1)}, \frac{\left(M_{a}\right)^{1-1 / q-\tau}}{\mathbf{c}}\right\} .
$$

We conclude this section by presenting a couple of concrete examples of BVPs of the form (43) and satisfying assumptions (I)-(IV) introduced above. 
Example 1 Let $\varrho \in(0, \infty)$ be arbitrarily fixed and let $k: \Lambda \rightarrow \mathbb{R}$ be the (unique) 1-periodic prolongation (on the whole of $\mathbb{R}$ ) of the function

$$
k_{0}(t)=\sqrt{t(1-t)}, \quad 0 \leq t \leq 1 .
$$

We then consider the following BVP

$$
\left\{\begin{array}{l}
\left(\sinh \left(k(t) e^{x(t)} x^{\prime}(t)\right)\right)^{\prime}=-\frac{\left|x^{\prime}(t)\right|}{\varrho+\cos ^{2}(t)} \text { a.e. on } \Lambda, \\
x(0)=0, \quad x(\infty)=1 .
\end{array}\right.
$$

Obviously, the above problem takes the form (43) with

(*) $a: \Lambda \times \mathbb{R} \rightarrow \mathbb{R}, \quad a(t, x)=k(t) \cdot e^{x}$;

(*) $\Phi: \mathbb{R} \rightarrow \mathbb{R}, \quad \Phi(z)=\sinh (z)$;

(*) $f_{1}: \Lambda \times \mathbb{R} \rightarrow \mathbb{R}, \quad f_{1}(t):=-\left(\varrho+\cos ^{2}(t)\right)^{-1}$;

(*) $f_{2}: \mathbb{R} \rightarrow \mathbb{R}, \quad f_{2}(y):=|y|$.

We claim that the functions $a, \Phi, f_{1}$ and $f_{2}$ satisfy all assumptions (I)-(IV) introduced in this section, with suitable constants fulfilling (52).

(I) Clearly, $a$ is nonnegative and continuous on $\Lambda \times \mathbb{R}$; moreover, it is bounded on $\Lambda \times[0,1]$ (note that $a(t, x) \leq e$ for every $(t, x) \in \Lambda \times[0,1])$ and

$$
h(t):=\min _{x \in[0,1]} a(t, x)=k(t), \quad \text { for every } t \in \Lambda .
$$

Since $h \equiv k$ is 1 -periodic and since the function $k_{0}$ is integrable on [0,1], it is very easy to recognize that $1 / h \in L_{\text {loc }}^{p}(\Lambda)$ for every $p \in(1,2)$; on the other hand, for every such $p$ and every $\sigma>1$ we also have

$$
\begin{aligned}
\int_{1}^{\infty} \frac{1}{t^{\sigma} h(t)^{p}} \mathrm{~d} t & =\sum_{n=1}^{\infty} \int_{n}^{n+1} \frac{1}{t^{\sigma} h(t)^{p}} \mathrm{~d} t \\
& =\sum_{n=1}^{\infty} \int_{0}^{1} \frac{1}{(t+n)^{\sigma}(\sqrt{t(1-t)})^{p}} \mathrm{~d} t \\
& \leq\left(\int_{0}^{1} \frac{1}{(\sqrt{t(1-t)})^{p}} \mathrm{~d} t\right) \sum_{n=1}^{\infty} \frac{1}{n^{\sigma}}<\infty
\end{aligned}
$$

and thus $a$ fulfills assumption (I) (with any choice of $\sigma \in(1, \infty)$ ).

(II) It is straightforward to recognize that $\Phi$ is a strictly increasing homeomorphism from $\mathbb{R}$ into itself and that $\Phi$ is odd; moreover, since we have

$$
\lim _{z \rightarrow 0^{+}} \frac{\Phi(z)}{z}=1
$$

we conclude that $\Phi$ fulfills assumption (II) with the choice $\rho=1$.

(III) First of all, $f_{1}$ is a Carathéodory function on $\Lambda \times \mathbb{R}$ (as it is continuous on the same set); moreover, for every $t \geq 0$ and every $x \in \mathbb{R}$ we have

$$
\left|f_{1}(t, x)\right| \leq \frac{1}{\varrho}=: \lambda(t)
$$


and thus $f_{1}$ fulfills assumption (III) $)_{1}$. As for assumption (III) $)_{2}$, we observe that, for every $t \in \Lambda$ and every $x \in \mathbb{R}$, we have

$$
\frac{1}{\varrho+1} \leq\left|f_{1}(t, x)\right| \leq \frac{1}{\varrho}
$$

as a consequence, (47) holds true with the choice

$$
\delta_{1}=\delta_{2}=0 \quad \text { and } \quad T_{0}=1 .
$$

Finally, since $f_{1}(t, x)<0$ for every $t \geq 0$ and every $x \in \mathbb{R}$, we conclude that $f_{1}$ also fulfills assumption (III) 3 (again with $T_{0}=1$ ).

(IV) Obviously, $f_{2}(y)=|y|$ is continuous on the whole of $\mathbb{R}$ and it fulfills assumption $(\mathrm{IV})_{1}$; moreover, since we have

$$
\Phi(y)=y+o(y) \text { as } y \rightarrow 0,
$$

it is possible to find two constants $c_{2}, c_{2}^{\prime}>0$ and a real $y^{*}>0$ such that

$$
|\Phi(y)|<1 \text { and } c_{2}|\Phi(y) \leq| y\left|\leq c_{2}^{\prime}\right| \Phi(y) \mid \text {, for }|y| \leq y^{*} .
$$

From this, it is immediate to check that condition (48) is satisfied for every $\gamma_{1}>1$ and every $\gamma_{2} \leq 1$. We then make the following (ad hoc) choice

$$
\gamma_{2}:=1 ; \quad \gamma_{1} \in(1,2) \text {. }
$$

We now turn our attention to assumptions (IV) $)_{3}$ and (IV) $)_{4}$. As for the former, we observe that, since $f_{2}$ is homogeneous of degree 1 , identity (50) is satisfied with $\omega(s)=s$; moreover, since $p \in(1,2)$, we also have

$$
\liminf _{s \rightarrow 0^{+}} \frac{\omega(s)}{s^{p}}=\liminf _{s \rightarrow 0^{+}} s^{1-p}=\infty .
$$

As regards assumption (IV) $)_{4}$, instead, from the trivial inequality

$$
\Phi(y)=\sinh (y) \geq y, \quad \text { for every } y \geq 1,
$$

we immediately conclude that (51) is satisfied with the choice

$$
c_{3}^{\prime}=1, \quad H=1, \quad \alpha=1 .
$$

To prove that Theorem 1 can be applied to the BVP (63) considered in this example, we are then left to show that the relations in (52) hold true.

On the one hand, since $\delta_{1}=0$, from (65) we infer that

$$
\frac{\delta_{1}+1}{\gamma_{1}-1}=\frac{1}{\gamma_{1}-1}>1=\rho
$$

on the other hand, since $\delta_{2}=0$ and $\gamma_{2}=1$, again by (65) we get

$$
\frac{\gamma_{2}\left(\delta_{1}+1\right)}{\gamma_{1}-1}=\frac{1}{\gamma_{1}-1}>1=1+\delta_{2} \text {. }
$$

From this, since $\sigma$ can be chosen arbitrarily close to 1 , we conclude that both the relations in (52) are satisfied. We are then entitled to apply our Theorem 1, which ensures the existence of a solution $x \in W^{1, p}(\Lambda)$ of (63).

It should be noticed that, even with the choice

$$
a(t, x) \equiv h(t),
$$


problem (63) does not fall in the class of examples considered in [3]: This is due to the fact that (51) holds but $\Phi$ is not homogeneous (see [3, Remark 5.2]). The reason why we are able to cover also this case is that we require the Nagumo-Wintner condition to hold in the modified form (11) (cf. with [3, eq. (11)]).

Example 2 Let $n \geq 2$ be a natural number and let $\varrho \in(0, \infty)$. Moreover, let $r, \vartheta_{1}, \vartheta_{2} \in \mathbb{R}$ be three real numbers satisfying the following relations:

$$
1<r<n+1 \quad \text { and } \quad 0<\vartheta_{1}<\vartheta_{2} \leq r-1 .
$$

Finally, let $\Phi_{r}(z)=|z|^{r-2} z$ be the usual $r$-Laplacian on $\mathbb{R}$. We then consider the following problem boundary value problem on $\Lambda=[0, \infty)$ :

$$
\left\{\begin{array}{l}
{\left[\Phi_{r}\left(\left(\frac{\sqrt[n]{t}}{1+\sqrt[n]{t}}+\sin ^{2}(x(t))\right) x^{\prime}(t)\right)\right]^{\prime}=\left(1-t^{\varrho}\right) \max \left\{\left|x^{\prime}(t)\right|^{\vartheta_{1}},\left|x^{\prime}(t)\right|^{\vartheta_{2}}\right\},} \\
x(0)=0, \quad x(\infty)=1 .
\end{array}\right.
$$

Obviously, the above problem takes the form (43) with

$(*) a: \Lambda \times \mathbb{R} \rightarrow \mathbb{R}, \quad a(t, x)=\sqrt[n]{t} /(1+\sqrt[n]{t})+\sin ^{2}(x) ;$

(*) $\Phi: \mathbb{R} \rightarrow \mathbb{R}, \quad \Phi(z)=\Phi_{r}(z)=|z|^{r-2} z ;$

(*) $f_{1}: \Lambda \times \mathbb{R} \rightarrow \mathbb{R}, \quad f_{1}(t):=1-t^{\varrho}$

(*) $f_{2}: \mathbb{R} \rightarrow \mathbb{R}, \quad f_{2}(y):=\max \left\{|y|^{\vartheta_{1}},|y|^{\vartheta_{2}}\right\}$.

We claim that, also in this case, the functions $a, \Phi, f_{1}$ and $f_{2}$ satisfy all assumptions (I)-(IV) with suitable constants fulfilling (52).

(I) Clearly, $a$ is nonnegative and continuous on $\Lambda \times \mathbb{R}$; moreover, it is bounded on $\Lambda \times[0,1]$ (note that $a(t, x) \leq 2$ for every $(t, x) \in \Lambda \times[0,1])$ and

$$
h(t):=\min _{x \in[0,1]} a(t, x)=\frac{\sqrt[n]{t}}{1+\sqrt[n]{t}}, \quad \text { for every } t \in \Lambda .
$$

Now, it is immediate to see that $1 / h \in L_{\text {loc }}^{p}(\Lambda)$ for every $p \in(1, n)$; in particular, since $n \geq 2$ and $r-1<n$, we can make the choice

$$
p \in(\max \{1, r-1\}, n) \text {. }
$$

Furthermore, for every $\sigma>1$ we also have

$$
\int_{1}^{\infty} \frac{1}{t^{\sigma} h(t)^{p}} \mathrm{~d} t=\int_{1}^{\infty} \frac{1}{t^{\sigma}}\left(1+\frac{1}{\sqrt[n]{t}}\right)^{p} \mathrm{~d} t<\infty,
$$

and thus $a$ fulfills assumption (I) (with any choice of $\sigma \in(1, \infty)$ ).

(II) It is straightforward to recognize that $\Phi$ is a strictly increasing homeomorphism from $\mathbb{R}$ into itself and that $\Phi$ is odd; moreover, since we have

$$
\lim _{z \rightarrow 0^{+}} \frac{\Phi(z)}{z^{r-1}}=1
$$

we conclude that $\Phi$ fulfills assumption (II) with the choice $\rho=r-1$.

(III) First of all, $f_{1}$ is a Carathéodory function on $\Lambda \times \mathbb{R}$ (as it is continuous on the same set); moreover, for every $t \geq 0$ and every $x \in \mathbb{R}$ we have

$$
\left|f_{1}(t, x)\right|=t^{\varrho}-1 \leq t^{\varrho}=: \lambda(t),
$$


and thus $f_{1}$ fulfills assumption (III) 1 (note that $\varrho>0$ ). As for assumption (III) 2 , we observe that, for every $t \geq 2^{1 / \varrho}$ and every $x \in \mathbb{R}$, we have

$$
\frac{t^{\varrho}}{2} \leq\left|f_{1}(t, x)\right| \leq t^{\varrho}
$$

as a consequence, (47) holds true with the choice

$$
\delta_{1}=\delta_{2}=\varrho>0 \quad \text { and } \quad T_{0}=2^{1 / \varrho} .
$$

Finally, since $f_{1}(t, x)<0$ for every $t \geq 2^{1 / \varrho}$ and every $x \in \mathbb{R}$, we conclude that $f_{1}$ also fulfills assumption (III) 3 (again with $T_{0}=2^{1 / \varrho}$ ).

(IV) Obviously, $f_{2}(y)$ is continuous on the whole of $\mathbb{R}\left(\right.$ as $\left.\vartheta_{1}, \vartheta_{2}>0\right)$ and it fulfills assumption (IV) 1 ; moreover, since $\vartheta_{1}<\vartheta_{2}$ one has

$$
\left|f_{2}(y)\right|=|y|^{\vartheta_{1}}, \quad \text { for every } y \in \mathbb{R} \text { with }|y| \leq 1 .
$$

As a consequence, since we have

$$
|\Phi(y)|=|y|^{r-1} \quad \text { for every } y \in \mathbb{R},
$$

and since, by assumption $\vartheta_{1} /(r-1) \leq 1$ (see (66)), it is very easy to recognize condition (48) is satisfied for every $\gamma_{1}, \gamma_{2} \in \mathbb{R}$ with $\gamma_{1}>1$ and $\gamma_{2} \leq \vartheta_{1} /(r-1)$. We then make the following (ad hoc) choice

$$
\gamma_{2}:=\frac{\vartheta_{1}}{r-1} \leq 1 ; \quad \gamma_{1} \in\left(1, \min \left\{1+\frac{\vartheta_{1}}{r-1}, 1+\frac{1+\varrho}{r-1}\right\}\right) .
$$

We now turn our attention to assumptions (IV) $)_{3}$ and (IV) 4 . As for the former, we observe that, since $\vartheta_{1}<\vartheta_{2}$, identity (50) is satisfied with

$$
\omega(s)=\max \left\{s^{\vartheta_{1}}, s^{\vartheta_{2}}\right\}= \begin{cases}s^{\vartheta_{1}}, & \text { if } 0 \leq s \leq 1, \\ s^{\vartheta_{2}}, & \text { if } s>1 ;\end{cases}
$$

moreover, as $\vartheta_{1} \leq r-1<p$, we also have

$$
\liminf _{s \rightarrow 0^{+}} \frac{\omega(s)}{s^{p}}=\liminf _{s \rightarrow 0^{+}} s^{\vartheta_{1}-p}=\infty
$$

Finally, as regards assumption $(\mathrm{IV})_{4}$, since we have

$$
f_{2}(y)=|y|^{\vartheta_{2}} \text { for every } y \in \mathbb{R} \text { with }|y| \geq 1,
$$

and since, again by assumption, $\vartheta_{2} \leq r-1$ (see (66)), we immediately conclude that (51) is satisfied with the choice

$$
c_{3}^{\prime}=1, \quad H=1, \quad \alpha=1 .
$$

To prove that Theorem 1 can be applied to the BVP (67) considered in this example, we are then left to show that the relations in (52) hold true.

On the one hand, since $\delta_{1}=\varrho$, from (70) we infer that

$$
\frac{\delta_{1}+1}{\gamma_{1}-1}=\frac{\varrho+1}{\gamma_{1}-1}>r-1=\rho ;
$$

on the other hand, since $\delta_{2}=\varrho$ and $\gamma_{2}=\vartheta_{1} /(r-1)$, again by (70) we get

$$
\frac{\gamma_{2}\left(\delta_{1}+1\right)}{\gamma_{1}-1}=\frac{\vartheta_{1}}{r-1} \cdot \frac{\varrho+1}{\gamma_{1}-1}>\varrho+1=1+\delta_{2} .
$$


From this, since $\sigma$ can be chosen arbitrarily close to 1 , we conclude that both the relations in (52) are satisfied. We are then entitled to apply our Theorem 1, which ensures the existence of a solution $x \in W_{\text {loc }}^{1, p}(\Lambda)$ of (63).

We explicitly observe that, even with the choice

$$
a(t, x)=\frac{\sqrt[n]{t}}{1+\sqrt[n]{t}}
$$

also problem (67) does not fall in the class of examples considered in [3]: This is due to the fact that the function $f_{2}$ is not homogeneous.

Example 3 Let us consider the following BVP:

$$
\left\{\begin{array}{l}
\left(\sqrt[3]{\arctan (\sqrt{t}) \cosh (x(t)) x^{\prime}(t)}\right)^{\prime}=-\left(x^{2}+1\right)\left(1+\sqrt{t} \sin ^{2}(t)\right)\left|x^{\prime}(t)\right|^{1 / 2}, \\
x(0)=0, \quad x(\infty)=1 .
\end{array}\right.
$$

Obviously, the above problem takes the form (43) with

(*) $a: \Lambda \times \mathbb{R} \rightarrow \mathbb{R}, \quad a(t, x)=\arctan (\sqrt{t}) \cdot \cosh (x) ;$

(*) $\Phi: \mathbb{R} \rightarrow \mathbb{R}, \quad \Phi(z)=\sqrt[3]{z}$;

(*) $f_{1}: \Lambda \times \mathbb{R} \rightarrow \mathbb{R}, \quad f_{1}(t):=-\left(x^{2}+1\right)\left(1+\sqrt{t} \sin ^{2}(t)\right)$;

(*) $f_{2}: \mathbb{R} \rightarrow \mathbb{R}, \quad f_{2}(y):=|y|^{1 / 2}$.

We claim that, also in this case, the functions $a, \Phi, f_{1}$ and $f_{2}$ satisfy all assumptions (I)-(IV) with suitable constants fulfilling (52).

(I) Clearly, $a$ is nonnegative and continuous on $\Lambda \times \mathbb{R}$; moreover, it is bounded on $\Lambda \times[0,1]$ (note that $2 a(t, x) \leq \pi \cosh (1)$ for every $(t, x) \in \Lambda \times[0,1])$ and

$$
h(t):=\min _{x \in[0,1]} a(t, x)=\arctan (\sqrt{t}), \quad \text { for every } t \in \Lambda .
$$

Now, it is immediate to check that $1 / h \in L_{\text {loc }}^{p}(\Lambda)$ for every $p \in(1,2)$; furthermore, for every such $p$ and every $\sigma>1$ we also have

$$
\int_{1}^{\infty} \frac{1}{t^{\sigma} h(t)^{p}} \mathrm{~d} t \leq\left(\frac{4}{\pi}\right)^{p} \int_{1}^{\infty} \frac{1}{t^{\sigma}} \mathrm{d} t<\infty,
$$

whence $a$ fulfills assumption (I) (with any choice of $\sigma \in(1, \infty)$ ).

(II) It is straightforward to recognize that $\Phi$ is a strictly increasing homeomorphism from $\mathbb{R}$ into itself and that $\Phi$ is odd; moreover, since we have

$$
\lim _{z \rightarrow 0^{+}} \frac{\Phi(z)}{\sqrt[3]{z}}=1
$$

we conclude that $\Phi$ fulfills assumption (II) with the choice $\rho=1 / 3$.

(III) First of all, $f_{1}$ is a Carathéodory function on $\Lambda \times \mathbb{R}$ (as it is continuous on the same set); moreover, for every $t \geq 0$ and every $x \in \mathbb{R}$ we have

$$
\left|f_{1}(t, x)\right|=\left(x^{2}+1\right)\left(\sqrt{t} \sin ^{2}(t)+1\right) \leq 2(\sqrt{t}+1)=: \lambda(t),
$$

and thus $f_{1}$ fulfills assumption (III) $)_{1}$. As for assumption (III) $)_{2}$, we observe that, for every $t \geq 1$ and every $x \in[0,1]$, we have

$$
1 \leq\left|f_{1}(t, x)\right| \leq 4 \sqrt{t}
$$


as a consequence, (47) holds true with the choice

$$
\delta_{1}=0, \quad \delta_{2}=\frac{1}{2} \quad \text { and } \quad T_{0}=1 .
$$

Finally, since $f_{1}(t, x)<0$ for every $t \in \Lambda$ and every $x \in \mathbb{R}$, we conclude that $f_{1}$ also fulfills assumption (III) ${ }_{3}$ (again with $T_{0}=1$ ).

Obviously, $f_{2}(y)=|y|^{1 / 2}$ is continuous on the whole of $\mathbb{R}$ and it fulfills assumption (IV) ${ }_{1}$; moreover, since we have

$$
|\Phi(y)|^{3 / 2}=|y|^{1 / 2} \leq|y|^{1 / 3}=|\Phi(y)|, \quad \text { for every } y \in \mathbb{R} \text { with }|y| \leq 1,
$$

it is immediate to recognize that condition (48) is satisfied with

$$
\gamma_{2}:=1 ; \quad \gamma_{1}:=\frac{3}{2}
$$

We now turn our attention to assumptions (IV) 3 and (IV) $)_{4}$. As for the former, since $f_{2}$ is homogeneous of degree $1 / 2$, identity (50) holds with

$$
\omega(s):=\sqrt{s}
$$

as a consequence, we have

$$
\lim _{s \rightarrow 0^{+}} \frac{\omega(s)}{s^{1 / 2}}>0,
$$

and thus condition (49) holds in the stronger form (60) (with $\tau=1 / 2$ ). According to Remark 8, we can then prove that $f_{2}$ fulfills (IV) ${ }_{4}^{\prime}$ (instead of (IV) $)_{4}$ ) with some $q \geq 2$. On the other hand, since $f_{2}(y)=|y|^{1 / 2}$, we trivially see that (61) in assumption (IV) ${ }_{4}^{\prime}$ is satisfied with $q=2$.

To prove that Theorem 1 can be applied to the BVP (71) considered in this example, we are then left to show that the relations in (52) hold true.

On the one hand, since $\delta_{1}=0$, from (73) we infer that

$$
\frac{\delta_{1}+1}{\gamma_{1}-1}=2>\frac{1}{3}=\rho
$$

on the other hand, since $\delta_{2}=1 / 2$, again by (73) we get

$$
\frac{\gamma_{2}\left(\delta_{1}+1\right)}{\gamma_{1}-1}=2>\frac{3}{2}=1+\frac{1}{2}=1+\delta_{2} .
$$

From this, since $\sigma$ can be chosen arbitrarily close to 1 , we conclude that both the relations in (52) are satisfied. We are then entitled to apply our Theorem 1, which ensures the existence of a solution $x \in W_{\text {loc }}^{1, p}(\Lambda)$ of (71).

\section{References}

1. Bereanu, C., Mawhin, J.: Periodic solutions of nonlinear perturbations of $\Phi$-Laplacians with possibly bounded $\Phi$. Nonlinear Anal. Theory Methods Appl. 68, 1668-1681 (2008)

2. Biagi, S., Calamai, A., Papalini, F.: Existence results for boundary value problems associated with singular strongly nonlinear equations. Nonlinear Anal. (2018) (preprint)

3. Biagi, S., Calamai, A., Papalini, F.: Heteroclinic solutions for a class of boundary value problems associated with singular equations. Nonlinear Anal. 184, 44-68 (2019) 
4. Bobisud, L.: Steady-state turbulent flow with reaction. Rocky Mt. J. Math. 21, 993-1007 (1991)

5. Brezis, H.: Functional Analysis, Sobolev Spaces and Partial Differential Equations. Universitext. Springer, New York (2011)

6. Cabada, A.: An overview of the lower and upper solutions method with nonlinear boundary value conditions. Bound. Value Probl. 2011, 893753 (2011)

7. Cabada, A., Pouso, R.: Existence results for the problem $\left(\varphi\left(u^{\prime}\right)\right)^{\prime}=f\left(t, u, u^{\prime}\right)$ with periodic and Neumann boundary conditions. Nonlinear Anal. 30, 1733-1742 (1997)

8. Cabada, A., Pouso, R.: Existence results for the problem $\left(\varphi\left(u^{\prime}\right)\right)^{\prime}=f\left(t, u, u^{\prime}\right)$ with nonlinear boundary conditions. Nonlinear Anal. 35, 221-231 (1999)

9. Calamai, A.: Heteroclinic solutions of boundary value problems on the real line involving singular $\Phi$ Laplacian operators. J. Math. Anal. Appl. 378, 667-679 (2011)

10. Calamai, A., Marcelli, C., Papalini, F.: Boundary value problems for singular second order equations. Fixed Point Theory Appl. 2018, 20 (2018)

11. Cupini, G., Marcelli, C., Papalini, F.: Heteroclinic solutions of boundary-value problems on the real line involving general nonlinear differential operators. Differ. Integral Equ. 24, 619-644 (2011)

12. Cupini, G., Marcelli, C., Papalini, F.: On the solvability of a boundary value problem on the real line. Bound. Value Probl. 2011, 16 (2011)

13. Dang, H., Oppenheimer, S.: Existence and uniqueness results for some nonlinear boundary value problems. J. Math. Anal. Appl. 198, 35-48 (1996)

14. El Khattabi, N., Frigon, M., Ayyadi, N.: Multiple solutions of boundary value problems with $\phi$-Laplacian operators and under a Wintner-Nagumo growth condition. Bound. Value Probl. 2013, 236 (2013)

15. Esteban, J., Vazquez, J.: On the equation of turbulent filtration in one-dimensional porous media. Nonlinear Anal. 10, 1303-1325 (1986)

16. Ferracuti, L., Papalini, F.: Boundary-value problems for strongly non-linear multivalued equations involving different $\Phi$-Laplacians. Adv. Differ. Equ. 14, 541-566 (2009)

17. Franco, D., O'Regan, D.: A new upper and lower solutions approach for second order problems with non-linear boundary conditions. Arch. Inequal. Appl. 1, 413-419 (2003)

18. Garcia-Huidobro, M., Manásevich, R., Zanolin, F.: A Fredholm-like result for strongly nonlinear second order ODS's. J. Differ. Equ. 114, 132-167 (1994)

19. Garcia-Huidobro, M., Manásevich, R., Zanolin, F.: On a pseudo fučik spectrum for strongly nonlinear second order ods's and an existence result. J. Comput. Appl. Math. 52, 219-239 (1994)

20. Garcia-Huidobro, M., Manásevich, R., Zanolin, F.: Strongly nonlinear second-order ODE's with rapidly growing terms. J. Math. Anal. Appl. 202, 1-26 (1996)

21. Herrero, M., Vazquez, J.: On the propagation properties of a non linear degenerate parabolic equation. Commun. Partial Differ. Equ. 7, 1381-1402 (1982)

22. Liu, Y.: Multiple positive solutions to mixed boundary value problems for singular ordinary differential equations on the whole line. Nonlinear Anal. Model. Control 17, 460-480 (2012)

23. Liu, Y., Yang, P.: Existence and non-existence of positive solutions of BVPs for singular ODEs on whole lines. Kyungpook Math. J. 55, 997-1030 (2015)

24. Marcelli, C.: Existence of solutions to boundary-value problems governed by general non-autonomous nonlinear differential operators. Electron. J. Differ. Equ. 171, 1-18 (2012)

25. Marcelli, C.: The role of boundary data on the solvability of some equations involving non-autonomous nonlinear differential operators. Bound. Value Probl. 2013, 252 (2013)

26. Marcelli, C., Papalini, F.: Comparison results and existence of bounded solutions to strongly nonlinear second order differential equations. Topol. Methods Nonlinear Anal. 34, 91-110 (2009)

27. Marcelli, C., Papalini, F.: Boundary value problems for strongly nonlinear equations under a WintnerNagumo growth condition. Bound. Value Probl. 2017, 183 (2017)

28. O'Regan, D.: Existence theory for $\left(\varphi\left(y^{\prime}\right)\right)^{\prime}=q f\left(t, y, y^{\prime}\right), 0<t<1$. Commun. Appl. Anal. 1, 33-52 (1997)

29. Wang, J., Gao, W.: Existence of solutions to boundary value problems for a non-linear second order equation with weak Carathéodory functions. Differ. Equ. Dyn. Syst. 5, 175-185 (1997)

30. Wang, J., Gao, W., Lin, Z.: Boundary value problems for general second order equation and similarity solutions to the Rayleigh problem. Tohoku Math. J. 47, 327-344 (1995)

Publisher's Note Springer Nature remains neutral with regard to jurisdictional claims in published maps and institutional affiliations. 